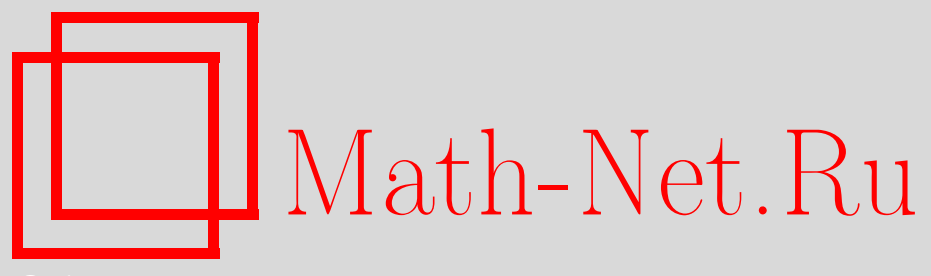

А. А. Карацуба, Арифметические проблемы теории характеров Дирихле, УМН, 2008, том 63, выпуск 4, 43-92

DOI: https://doi.org/10.4213/rm9234

Использование Общероссийского математического портала Math-Net.Ru подразумевает, что вы прочитали и согласны с пользовательским соглашением http://www . mathnet.ru/rus/agreement

Параметры загрузки:

IP: 34.239 .49 .27

26 апреля 2023 г., 18:20:11

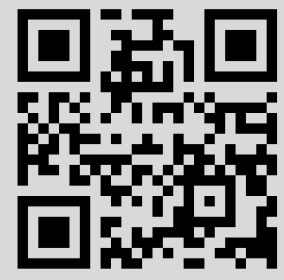


УДК 511

\title{
Арифметические проблемы теории характеров Дирихле
}

\author{
А. А. Карацуба \\ Дается обзор результатов, связанных с распределением значений ха- \\ рактеров Дирихле на редких множествах натурального ряда чисел. \\ Библиография: 99 названий.
}

\section{СОДЕРЖАНИЕ}

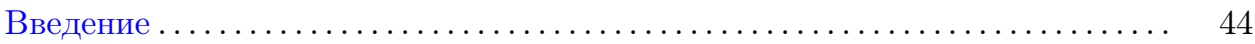

Глава І. Вспомогательные утверждения . . . . . . . . . . . . . . . . . 54

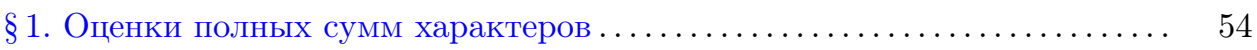

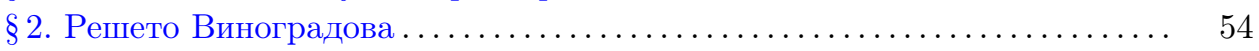

Глава II. Линейные суммы характеров с простыми числами . . . . . . . . . 56

$\S 1$. Суммы по подряд идущим простым числам ................... 56

$\S 2$. Суммы по простым числам, принадлежащим арифметической прогрес-

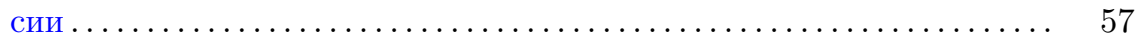

§3. Распределение произведений сдвинутых простых чисел в коротких

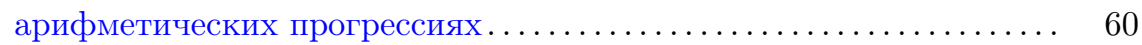

Глава III. Нелинейные суммы характеров с простыми числами . . . . . . . 64

$\S 1$. Суммы по подряд идущим простым числам ................ 64

$\S 2$. Суммы по простым числам, принадлежащим арифметической прогрессии ........................................... 65

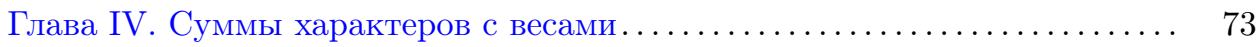

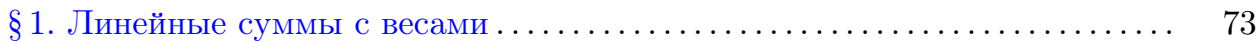

$\S 2$. Нелинейные суммы символов Лежандра с весами ............... 76

$\S 3$. Нелинейные суммы характеров с весами .................. 76

Глава V. Распределение значений характеров Дирихле на аддитивных последовательностях . . . . . . . . . . . . . . . . . . . 78

$\S 1$. Распределение степенных вычетов и невычетов в аддитивных после-

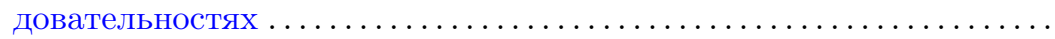

$\S 2$. Распределение значений характеров Дирихле на аддитивных последовательностях ....................................... 80

Глава VI. Некоторые специальные вопросы теории характеров, нерешен-

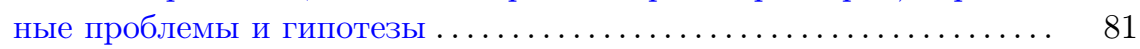

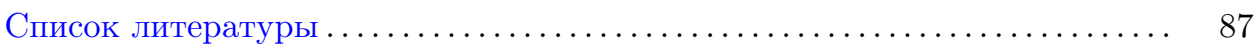

(C) А. А. КАРАцУБА, 2008 


\section{Введение}

При доказательстве теоремы о бесконечности множества простых чисел, лежащих в арифметической прогрессии, первый член которой взаимно прост с разностью прогрессии, Л. Дирихле ввел функцию целочисленного аргумента, которая позднее стала называться характером Дирихле и которая нашла многочисленные применения в математике. Есть два способа определения характера Дирихле, именно, аксиоматический и конструктивный. Начнем с первого, хотя сам Дирихле определял характеры конструктивно (см., например, [1; с. 296-309]). Везде ниже часто вместо "характер Дирихле" будем говорить "характер".

ОПРедЕлЕниЕ. Характером называется комплекснозначная функция $\chi(n)$, областью определения которой является множество целых чисел $\mathbb{Z}$, удовлетворяющая следующим условиям:

1) $\chi(1)=1$

2) $\chi(n)$ - вполне мультипликативная функция, т.е. для любых $n, m \in \mathbb{Z}$ выполняется равенство

$$
\chi(n m)=\chi(n) \chi(m)
$$

3) существует $k \in \mathbb{Z}, k \geqslant 1$, такое, что $\chi(n)$ - периодическая функция с периодом $k$, т. е. для любого $n \in \mathbb{Z}$ выполняется равенство

$$
\chi(n+k)=\chi(n) .
$$

Легко доказать, что все периоды $\chi(n)$ кратны наименьшему периоду $k \geqslant 1$, который называется главным периодом. Число $k$ называется также основным модулем $\chi(n)$ или просто модулем $\chi(n)$, а $\chi(n)$ - характером по модулю $k$. Иногда вместо $\chi(n)$ пишут $\chi(n ; k)$. Везде ниже модуль характера будет основным.

Пусть $\chi(n)$ - характер по модулю $k$ и $\varphi(k)$ - функция Эйлера $(\varphi(k)$ равняется количеству натуральных чисел, не превосходящих $k$ и взаимно простых с $k$ ). Из определения характера и теоремы Эйлера следует, что если $(n, k)=1$, то

$$
1=\chi\left(n^{\varphi(k)}\right)=(\chi(n))^{\varphi(k)},
$$

т. е. $\chi(n)$ есть корень степени $\varphi(k)$ из единицы. Кроме того, если $(n, k)=d>1$, то $\chi(n)=0$. Действительно, в этом случае $k=k_{1} d, 1 \leqslant k_{1}<k$, так что $k_{1}$ не является периодом $\chi(n)$. Поэтому найдется такое $a \in \mathbb{Z}$, что

$$
\chi\left(a+k_{1}\right) \neq \chi(a), \quad \lambda=\chi\left(a+k_{1}\right)-\chi(a) \neq 0 .
$$

Следовательно,

$$
\lambda \chi(d)=\chi\left(a d+k_{1} d\right)-\chi(a d)=\chi(a d+k)-\chi(a d)=\chi(a d)-\chi(a d)=0,
$$

т. е. $\chi(d)=0, \chi(n)=\chi\left(n_{1} d\right)=\chi\left(n_{1}\right) \chi(d)=0$.

Главным характером по модулю $k$ называется характер $\chi(n)$ такой, что

$$
\chi(n)= \begin{cases}1, & \text { если }(n, k)=1 \\ 0, & \text { если }(n, k)>1\end{cases}
$$


Обычно главный характер обозначается символом $\chi_{0}(n)$. Из (1) и определения $\chi_{0}(n)$ получаем следующее равенство:

$$
(\chi(n))^{\varphi(k)}=\chi_{0}(n) .
$$

Наименьшее натуральное число $m$ такое, что

$$
(\chi(n))^{m}=\chi_{0}(n),
$$

называется степенъю характера $\chi(n)$.

По модулю $k$ существует ровно $\varphi(k)$ разных характеров. Комплексно-сопряженный к $\chi(n)$ характер обозначается символом $\overline{\chi(n)}$.

Характеры обладают свойством "ортогональности", которое необходимо было Дирихле при доказательстве теоремы о простых числах в арифметической прогрессии:

$$
\frac{1}{\varphi(k)} \sum_{\chi \bmod k} \chi(n)=\left\{\begin{array}{lll}
1, & \text { если } n \equiv 1 & (\bmod k) \\
0, & \text { если } n \neq 1 & (\bmod k)
\end{array}\right.
$$

В последней сумме суммирование ведется по всем $\varphi(k)$ характерам по модулю $k$. Пользуясь этой формулой, можно аналитически выделять целые числа $n$, принадлежащие арифметической прогрессии вида $n \equiv l(\bmod k),(l, k)=1$. Действительно, полагая $l_{1} l \equiv 1(\bmod k), 1 \leqslant l_{1}<k$, находим:

$$
\delta_{0}(n)=\frac{1}{\varphi(k)} \sum_{\chi \bmod k} \chi(n) \chi\left(l_{1}\right)= \begin{cases}1, & \text { если } n \equiv l(\bmod k) ; \\ 0, & \text { если } n \neq l(\bmod k) .\end{cases}
$$

Следующая формула является “двойственной” к (2):

$$
\frac{1}{\varphi(k)} \sum_{n=1}^{k} \chi(n)= \begin{cases}1, & \text { если } \chi=\chi_{0} \\ 0, & \text { если } \chi \neq \chi_{0} .\end{cases}
$$

Теория характеров Дирихле, а также их применения в различных разделах математики хорошо известны (см., например, [1]-[10]). Меньше известны чисто арифметические проблемы теории характеров, которым и посвящена настоящая статья.

Арифметические проблемы связаны, в основном, с характерами, модуль $k$ которых равен простому числу. Везде далее будем считать $q$ нечетным простым числом и $\chi(n)$ - характером Дирихле по модулю $q$. Нетрудно понять, что $\chi(n)$ можно определить также следующими соотношениями (конструктивное определение $\chi(n))$ :

$$
\chi(n)= \begin{cases}0, & \text { если } n \equiv 0(\bmod q) ; \\ e^{2 \pi i \frac{a \text { ind } n}{q-1}}, & \text { если } n \neq 0(\bmod q) .\end{cases}
$$

Здесь $a$ - целое число, $0 \leqslant a<q-1$, ind $n$ - индекс числа $n$ по модулю $q$ при основании $g$, где $g$ - наименьший первообразный корень по модулю $q$. При $a=0$ получаем главный характер по модулю $q$, при $a=1, \ldots, q-2$ получаем 
$\varphi(q)-1$ разных неглавных характеров по модулю $q$. В частности, при $a=$ $\frac{q-1}{2}$ получаем характер, который называется символом Лежандра и имеет специальное обозначение $\left(\frac{n}{q}\right)$ :

$$
\left(\frac{n}{q}\right)=\left\{\begin{aligned}
1, & \text { если } n-\text { квадратичный вычет по модулю } q ; \\
-1, & \text { если } n-\text { квадратичный невычет по модулю } q ; \\
0, & \text { если } n \equiv 0(\bmod q) .
\end{aligned}\right.
$$

Заметим, что каждый характер по модулю $q$, определенный ранее аксиоматически, совпадает с функцией $\chi(n)$ формулы (3) при некотором $a, 0 \leqslant a<q-1$.

Наиболее важными арифметическими проблемами, которые связаны с характерами, являются проблемы распределения степенных вычетов и невычетов по заданному модулю и проблемы распределения первообразных корней по заданному модулю. Пусть $n$ - делитель $q-1$. Число $a$, не кратное $q$, называется вычетом степени $n$ по модулю $q$, если существует $b$ такое, что

$$
a \equiv b^{n}(\bmod q) .
$$

В противном случае $a$ называется невычетом степени $n$ по модулю $q$. При $n=2$ вычеты (невычеты) называются квадратичными, при $n=3$ - кубическими, при $n=4$ - биквадратичными. Натуральное число $g$ называется первообразным корнем по модулю $q$, если все $q-1$ чисел $1=g^{0}, g^{1}, g^{2}, \ldots, g^{q-2}$ попарно несравнимы по модулю $q$, т. е.

$$
g^{r} \not \equiv g^{s}(\bmod q), \quad 0 \leqslant r, s \leqslant q-2, \quad r \neq s .
$$

Первые вопросы о распределении квадратичных вычетов и невычетов поставлены Эйлером и Гауссом; им же принадлежат и первые теоремы, отвечающие на эти вопросы, в частности теорема о том, что в приведенной системе вычетов по модулю $q$ содержится $\frac{q-1}{2}$ квадратичных вычетов и $\frac{q-1}{2}$ квадратичных невычетов.

Вопрос о квадратичных вычетах и невычетах в подмножествах $A$ множества приведенной системы вычетов по модулю $q$ легко сводится к вопросу о верхней грани модуля суммы символов Лежандра. Действительно, пользуясь определением символа Лежандра, находим:

$$
\delta(n)=\frac{1}{2}\left(1+\left(\frac{n}{q}\right)\right)= \begin{cases}1, & \text { если } n-\text { квадратичный вычет по модулю } q ; \\ 0, & \text { если } n-\text { квадратичный невычет по модулю } q ; \\ \frac{1}{2}, & \text { если } n \equiv 0(\bmod q) .\end{cases}
$$

Поэтому, если $A \subseteq[1, q-1],\|A\|$ - количество чисел множества $A, V(A)$ и $N(A)$ - соответственно число квадратичных вычетов и число квадратичных невычетов в множестве $A$, то получаем:

$$
\begin{aligned}
& V(A)=\sum_{n \in A} \delta(n)=\frac{1}{2}\|A\|+\frac{1}{2} S(A), \\
& N(A)=\frac{1}{2}\|A\|-\frac{1}{2} S(A),
\end{aligned}
$$


где

$$
S(A)=\sum_{n \in A}\left(\frac{n}{q}\right) .
$$

Следовательно, если доказать, что

$$
|S(A)| \leqslant\|A\| \Delta, \quad 0<\Delta<1,
$$

то формулы (4), (5) станут асимптотическими с главным членом $\frac{1}{2}\|A\|$ и остатком, абсолютная величина которого не превосходит $\frac{1}{2}\|A\| \Delta$.

Точно так же решается задача о степенных вычетах по модулю $q$ произвольной степени $n \geqslant 2, n \mid(q-1)$. Действительно, число $a,(a, q)=1$, является вычетом степени $n$ по модулю $q$, если

$$
a \equiv x^{n}(\bmod q)
$$

Отсюда следует, что

$$
\text { ind } a \equiv n \text { ind } x(\bmod (q-1)),
$$

и так как $n \mid(q-1)$, то

$$
\text { ind } a \equiv 0(\bmod n) \text {. }
$$

Соотношение (6) является необходимым и достаточным условием для того, чтобы $a$ было вычетом степени $n$ по модулю $q$. Пусть $a \in A$ и $V_{n}(A)$ - количество тех $a$, которые являются вычетами степени $n$ по модулю $q$. Тогда, пользуясь (6) и свойством полной линейной тригонометрической суммы, находим:

$$
\begin{aligned}
V_{n}(A) & =\sum_{a \in A} \frac{1}{n} \sum_{b=0}^{n-1} e^{2 \pi i \frac{b \text { ind } a}{n}}=\frac{1}{n}\|A\|+\frac{1}{n} \sum_{b=1}^{n-1} \sum_{a \in A} e^{2 \pi i \frac{b \text { ind } a}{n}} \\
& =\frac{1}{n}\|A\|+\frac{1}{n} \sum_{b=1}^{n-1} \sum_{a \in A} \chi(a),
\end{aligned}
$$

где

$$
\chi(a)=\chi_{b}(a)=e^{2 \pi i \frac{b \text { ind } a}{n}}=e^{2 \pi i \frac{b_{1} \text { ind } a}{q-1}},
$$

$0<b_{1}=b(q-1) / n<q-1$, т. е. $\chi(a)-$ неглавный характер по модулю $q$. Если доказать, что

$$
\max _{\chi \neq \chi_{0}}\left|\sum_{a \in A} \chi(a)\right| \leqslant\|A\| \Delta,
$$

то из (7) будет следовать формула

$$
V_{n}(A)=\frac{1}{n}\|A\|+\frac{n-1}{n} \theta\|A\| \Delta, \quad|\theta| \leqslant 1,
$$

которая будет асимптотической, если

$$
(n-1) \Delta<1 \text {. }
$$


Подобным образом решается задача о количестве первообразных корней в $A$.

Для того чтобы натуральное число $а$ было первообразным корнем по модулю $q$, необходимо и достаточно выполнение следующего соотношения:

$$
(\text { ind } a, q-1)=1 \text {. }
$$

Поэтому, если $P(A)$ - количество первообразных корней в $A \subseteq[1, q-1]$, то, пользуясь известным свойством функции Мёбиуса $\mu(d)$ и свойством полной линейной тригонометрической суммы, последовательно находим:

$$
\begin{aligned}
P(A) & =\sum_{\substack{a \in A \\
(\text { ind } a, q-1)=1}} 1=\sum_{a \in A} \sum_{d \mid(\text { ind } a, q-1)} \mu(d) \\
& =\sum_{d \mid(q-1)} \mu(d) \quad \sum_{\substack{a \in A \\
a \equiv 0(\bmod d)}} 1=\sum_{d \mid(q-1)} \mu(d) \sum_{a \in A} \frac{1}{d} \sum_{b=0}^{d-1} e^{2 \pi i \frac{b \text { ind } a}{d}} \\
& =\sum_{d \mid(q-1)} \mu(d) \sum_{a \in A}\left(\frac{1}{d}+\frac{1}{d} \sum_{b=1}^{d-1} \chi_{b}(a)\right)
\end{aligned}
$$

где $\chi_{b}(a)=e^{2 \pi i \frac{b \text { ind } a}{d}}-$ неглавный характер по модулю $q$.

Следовательно, для $P(A)$ получаем формулу:

$$
P(A)=\|A\| \sum_{d \mid(q-1)} \frac{\mu(d)}{d}+\sum_{d \mid(q-1)} \frac{\mu(d)}{d} \sum_{b=1}^{d-1} \sum_{a \in A} \chi_{b}(a) .
$$

Далее имеем:

$$
\sum_{d \mid(q-1)} \frac{\mu(d)}{d}=\frac{\varphi(q-1)}{q-1}
$$

Если $q-1=q_{1}^{\alpha_{1}} \cdots q_{k}^{\alpha_{k}}-$ каноническое разложение $q-1$ на простые сомножители, то

$$
\sum_{d \mid(q-1)}|\mu(d)|=1+C_{k}^{1}+\cdots+C_{k}^{k}=2^{k} .
$$

Тем самым,

$$
P(A)=\frac{\varphi(q-1)}{q-1}\|A\|+\theta \cdot 2^{k}\|A\| \Delta,
$$

где $|\theta| \leqslant 1$ и $\max _{\chi \neq \chi_{0}}\left|\sum_{a \in A} \chi(a)\right|=\|A\| \Delta$. Так как при любом фиксированном $\varepsilon>0$ и $q \geqslant q_{1}(\varepsilon)$ выполняется неравенство

$$
k \leqslant(1+\varepsilon) \frac{\ln q}{\ln \ln q},
$$

и, кроме того,

$$
\frac{\varphi(q-1)}{q-1} \geqslant \frac{c}{\ln \ln q}, \quad c>0
$$


то формула (9) будет асимптотической, если

$$
\Delta \leqslant 2^{-(1+\varepsilon) \frac{\ln q}{\ln \ln q}}, \quad q \geqslant q_{2}(\varepsilon) .
$$

Итак, в приведенных задачах достаточно получить оценку вида

$$
\max _{\chi \neq \chi_{0}}\left|\sum_{a \in A} \chi(a)\right| \leqslant\|A\| \Delta
$$

с соответствующим $\Delta$, чтобы формулы (4), (5), (8), (9) были асимптотическими. Поэтому основной проблемой в арифметических приложениях характеров Дирихле является проблема получения оценки (10) с возможно меньшим $\Delta$. Оценки вида (10) можно трактовать также как ответ на вопрос о распределении значений характеров на множествах целых чисел той или иной арифметической природы (на множествах $A$ ).

Частным видом суммы, стоящей в левой части (10), является следующая:

$$
S=S(f ; X)=\sum_{x=1}^{X} \chi(f(x)) .
$$

Здесь $\chi$ - неглавный характер по модулю $q, X$ - натуральное число, $f(x)$ целозначная функция.

Так как тривиально $|S| \leqslant X$, то, тем самым, основной проблемой в рассматриваемых вопросах является проблема получения оценок вида

$$
|S| \leqslant X \Delta
$$

с возможно меньшим количеством слагаемых в $S$, т. е. с возможно меньшим $X$ по отношению к $q$, и с возможно меньшим $\Delta \leqslant 1$. Именно в такой форме мы будем рассматривать оценки сумм характеров в этой статье.

Если $\chi$ - символ Лежандра, $f(x)=x+a, X=q$, то из упомянутой выше теоремы Эйлера-Гаусса (с. 46) получаем:

$$
S=0 .
$$

Сумму $S=S(f ; X)$ с $f(x)=x+a$ далее будем обозначать символом $S_{0}(X)$. В 1918 г. И. М. Виноградов [11], [12] и независимо Г. Пойя [13] доказали, что

$$
\left|S_{0}(X)\right|<\sqrt{q} \ln q .
$$

Позднее неравенства, подобные (13), с многочленом $f(x)$ произвольной степени $n, f(x) \not \equiv g^{m}(x)(\bmod q)$, где $m$ - степень характера $\chi, X=q$, были доказаны Д. Морделлом, Г. Хассе, А. Вейлем. Окончательным результатом этого направления исследований явилась оценка Хассе-Вейля:

$$
\left|\sum_{x=1}^{q} \chi(f(x))\right| \leqslant(n-1) \sqrt{q} .
$$

Все упомянутые оценки были перенесены Г. Дэвенпортом [14] на суммы характеров в конечных полях произвольной фиксированной степени. Замечу также, 
что оценки вида (14) были доказаны Л. Карлицом и С. Учиямой [15] для аддитивного характера $\chi=\psi$ и рациональной функции $f(x)=x+a / x$ и Г. И. Перельмутером [16] для сумм слагаемых вида $\chi(R(x)) \psi(\Phi(x))$, где $R(x)$ и $\Phi(x)-$ рациональные функции. Из результатов последней работы, в частности, следует оценка неполной суммы характеров вида

$$
\left|\sum_{x=1}^{X} \chi(f(x))\right| \leqslant n \sqrt{q} \ln q .
$$

Здесь, как и выше, $f(x) \not \equiv g^{m}(x)(\bmod q), 1 \leqslant X<q$. Более подробные сведения об оценках полных сумм характеров, т. е. сумм, в которых переменная суммирования пробегает полную систему вычетов по модулю $q$, можно найти в [6].

В 1938 г. И. М. Виноградов стал рассматривать суммы $S$, в которых суммирование ведется по простым числам. Пользуясь созданным им методом, которым он решил проблему Гольдбаха, Виноградов [17] получил следующую оценку:

$$
\left|S_{1}(X)\right|=\left|\sum_{p \leqslant X} \chi(p+a)\right| \ll X^{1+\varepsilon} \sqrt{\frac{1}{q}+\frac{q}{\sqrt[3]{X}}} .
$$

Здесь $(a, q)=1, \varepsilon-$ произвольное положительное число, постоянная в знаке « зависит только от $\varepsilon$. Оценка (15) будет нетривиальной, если $X \geqslant q^{3+\varepsilon^{\prime}}, \varepsilon^{\prime}>0-$ любое фиксированное число. В 1943 г. Виноградов [18] уточнил (15) и, кроме того, оценил нелинейную сумму характеров с простыми числами, доказав, что

$$
\begin{array}{r}
\left|S_{1}(X)\right|=\left|\sum_{p \leqslant X} \chi(p+a)\right| \ll X^{1+\varepsilon} G, \\
\left|S_{2}(X)\right|=\left|\sum_{p \leqslant X} \chi(p(p+a))\right| \ll X^{1+\varepsilon} G,
\end{array}
$$

где

$$
G=\sqrt{\frac{1}{q}+\frac{q}{X}}+X^{-\frac{1}{6}}
$$

Последние оценки будут нетривиальными, если $X \geqslant q^{1+\varepsilon^{\prime}}$. Отметим, что оценки (15)-(17) представляют исключительный интерес, так как мало что известно даже о распределении простых чисел $p$ в коротких арифметических прогрессиях, т. е. в прогрессиях вида $p \equiv l(\bmod q),(l, q)=1, p \leqslant X \leqslant q^{A}, A-$ фиксированное положительное число.

В 1952 г. И. М. Виноградов [19] доказал, что

$$
\left|S_{1}(X)\right| \ll X^{1+\varepsilon}\left(q^{\frac{3}{4}} X^{-1}\right)^{\frac{1}{4}} .
$$

Из этой оценки видно, что она становится нетривиальной, если $X \geqslant q^{0.75+\varepsilon^{\prime}}$. Это совершенно удивительный результат. Если к указанной проблеме применять аналитический метод Римана, то естественно ожидать нетривиальной 
оценки $\left|S_{1}(X)\right|$ в том случае, когда в распределении простых чисел $p$ в арифметических прогрессиях с разностью $q$ наступит "порядок", а это будет, самое лучшее, при $X \geqslant q^{2+\varepsilon}$, так как из расширенной гипотезы Римана следует, что

$$
\pi(X ; q, l)=\frac{\pi(X)}{q-1}+O\left(X^{0.5+\varepsilon}\right),
$$

T. e.

$$
\pi(X ; q, l) \sim \frac{\pi(X)}{q-1} \quad \text { при } \quad X \geqslant q^{2+\varepsilon^{\prime}} .
$$

Правда, если $S_{1}$ представить в виде суммы по нулям соответствующих $L$-функций Дирихле и только потом воспользоваться расширенной гипотезой Римана, то тогда получится оценка $\left|S_{1}\right|$, нетривиальная уже при $X \geqslant q^{1+\varepsilon^{\prime}}$, т. е. упомянутый выше результат Виноградова 1943 г.

Казалось, что получилось то, чего не может быть. Ю.В. Линник в 1971 г. писал по этому поводу: “Эта оценка имеет принципиальное значение, так как по глубине превосходит то, что дает непосредственное применение расширенной гипотезы Римана, и, по-видимому, в этом направлении является истиной более глубокой, чем указанная гипотеза (если гипотеза верна)" (см. [20; с. 29]). С работой [19] связан еще один, отмеченный только в [21], факт. Если применить основную идею этой работы к оценке $\left|S_{0}(X)\right|$, то нетривиальный результат получится уже при $X \geqslant q^{0.375+\varepsilon}$.

В 1953 г. И. М. Виноградов [22] уточнил (18), доказав, что

$$
\left|S_{1}(X)\right| \ll X^{1+\varepsilon}\left(\left(q^{\frac{3}{4}} X^{-1}\right)^{\frac{1}{3}}+X^{-0.1}\right) .
$$

Работы Виноградова по оценкам сумм характеров с простыми числами были продолжены Г. И. Перельмутером [23], который нетривиально оценил нелинейные суммы самого общего вида при числе слагаемых $X$ большем, чем $q^{1+\varepsilon}$.

В 1957 г. Д. Берджесс [24] нетривиально оценил $\left|S_{0}(X)\right|$ при $X \geqslant q^{0.25+\varepsilon}$ (см. также [25]-[28]). Метод Д. Берджесса был применен Г. Дэвенпортом и Д. Льюисом [29] к оценкам сумм характеров в конечных полях степени $n$; они нетривиально оценили такие суммы при условии, что каждая координата переменной суммирования принимает $H$ последовательных значений целых чисел, где

$$
H>q^{\frac{n}{2 n+2}+\varepsilon} .
$$

С ростом $n$ результат теоремы ухудшается и стремится к старому результату Дэвенпорта [14]. Этой же задаче посвящена работа Берджесса [28], в которой при $n \geqslant 3$ получен аналогичный результат; при $n=2$ здесь же получена граница для $H$, подобная его границе для полей первой степени, именно, $H>q^{0.25+\varepsilon}$.

В 1968 г. автором [30] был разработан новый метод, который позволил получить нетривиальную оценку суммы характеров в конечных полях произвольной фиксированной степени $n \geqslant 1$ при $H>q^{0.25+\varepsilon}$ (см. также [31]). Развивая свой метод и соединяя его с методом Виноградова, автор [32], [33] нетривиально оценил $\left|S_{1}(X)\right|$ при $X \geqslant q^{0.5+\varepsilon}$. Наконец, в 1978 г. удалось нетривиально оценить и нелинейные суммы символов Лежандра с простыми числами типа $S_{2}(X)$ при $X \geqslant q^{0.75+\varepsilon}($ см. [34], [35]). 
Идея метода работы [30] такова. Рассмотрим двойную сумму

$$
W=\sum_{x}^{X} \sum_{y}^{Y} \chi(x+y)
$$

где $x$ и $y$ пробегают произвольные множества из $X$ и, соответственно, $Y$ различных по модулю $q$ чисел. Если $Y \geqslant q^{\varepsilon}, X \geqslant q^{0.5+\varepsilon}$, то для $|W|$ можно получить нетривиальную оценку вида

$$
|W| \ll X Y q^{-\delta}
$$

где $\delta=\delta(\varepsilon)>0$, а постоянная в знаке « зависит только от $\varepsilon$. Действительно, выбирая подходящее натуральное число $k=k(\varepsilon)$ и применяя оценку Хассе-Вейля (14), найдем:

$$
\begin{aligned}
|W|^{2 k} & \ll X^{2 k-1} \sum_{x=1}^{q}\left|\sum_{y}^{Y} \chi(x+y)\right|^{2 k} \\
& \ll X^{2 k-1}\left(q Y^{k}+\sqrt{q} Y^{2 k}\right)=(X Y)^{2 k} \Delta,
\end{aligned}
$$

где

$$
\Delta=\frac{q}{X Y^{k}}+\frac{\sqrt{q}}{X}
$$

Отсюда следует указанная оценка $|W|$.

Во многих задачах вместо условия $X \geqslant q^{0.5+\varepsilon}$ выполняются условия $X Y \geqslant$ $q^{0.5+\varepsilon}, X \geqslant q^{\varepsilon}, Y \geqslant q^{\varepsilon}$ и известно, что переменная $y$ пробегает сплошной промежуток суммирования. Прямо к $W$ предыдущие рассуждения применить нельзя, например, если $X=q^{3 / 8+\varepsilon}, Y=q^{1 / 8+\varepsilon}$. Однако, пользуясь тем, что интервал суммирования по $y$ сплошной, можно в сумме $W$ по $y$ сделать сдвиг вида $y \rightarrow y+u v$, где $u$ и $v$ принимают соответственно $U=Y q^{-\varepsilon_{1}}$ и $V=q^{\varepsilon_{2}}$ значений, $\varepsilon>\varepsilon_{1}>\varepsilon_{2}>0$. Тогда получим

$$
W \leqslant Y(U V)^{-1}\left|W_{1}\right|+O\left(X Y q^{-\varepsilon_{1}+\varepsilon_{2}}\right),
$$

где

$$
W_{1}=\sum_{x}^{X} \sum_{u=1}^{U} \sum_{v=1}^{V} \chi(x+u v)
$$

Далее находим:

$$
\left|W_{1}\right| \ll \sum_{x}^{X} \sum_{u=1}^{U}\left|\sum_{v=1}^{V} \chi\left(x u^{-1}+v\right)\right| \ll \sum_{z}^{Z} c(z)\left|\sum_{v=1}^{V} \chi(z+v)\right| .
$$

Здесь уже переменная $z$ принимает $Z$ значений,

$$
Z \asymp X U \geqslant q^{0.5+\varepsilon-\varepsilon_{1}},
$$

и можно применять вышеизложенные соображения, которые приводят к оценке (19). Заметим, что очень точное определение понятия "метод” дали Д. Пойя и Г. Сегё (см. [36; с. 11]): "Идея, примененная однажды, порождает искусственный прием, примененная дважды, она становится методом". 
Новый метод позволил получить целый ряд новых результатов и, прежде всего, в оценках сумм характеров, определенных на редких последовательностях натурального ряда чисел. Еще в 1966 г. И. М. Виноградов [37] оценил сумму типа $S_{1}(X)$, но с условием, что простые числа, по которым идет суммирование, принадлежат арифметической прогрессии. Продолжением этих исследований явились работы [38], [39], [21], где получены более точные и более общие результаты. С 1971 г. автор стал изучать распределение значений характеров на аддитивных последовательностях (см. [40], [41]). Первые результаты в этом направлении получены Г. Дэвенпортом и П. Эрдёшем [42] и П. Эрдёшем и Н. Шапиро [43]. Теорема Дэвенпорта-Эрдёша будет приведена в гл. I. Теорема Эрдёша-Шапиро такова: справедливо неравенство

$$
\left|\sum_{u \in S} \sum_{v \in T} \chi(u+v)\right| \leqslant \sqrt{q} \sqrt{\|S\|\|T\|},
$$

где $\|S\| u\|T\|$ - количество чисел в $S$ и $T$ соответственно.

И, наконец, несколько необычными выглядят работы по оценкам сумм характеров с весами и их арифметические приложения (см. [44]-[46]). Здесь также главную роль играет метод работы [30] и специфика рассматриваемых сумм.

Заканчивая введение, хочу подчеркнуть, что применения метода из [30], а также технических приемов из [32], [33], [38], [40] к решению ряда задач теории чисел помещены в учебнике [47] в качестве упражнений (см. также [5], [48], [4], [49]) и в настоящее время часто используются в исследованиях по теории характеров и их приложениям.

ОБОЗНАЧЕНИЯ. На протяжении всей статьи будем считать, что $q$ - нечетное простое число, $p$ - простое число; $\varepsilon, \varepsilon^{\prime}, \varepsilon_{1}, \ldots$ - произвольные положительные числа, не превосходящие 0.01, в разных утверждениях, вообще говоря, разные; $\varphi(n)$ - функция Эйлера, $\mu(n)$ - функция Мёбиуса, $\Lambda(n)$ - функция Мангольдта, $\tau_{k}(n)$ - число решений в натуральных числах $n_{1}, \ldots, n_{k}$ уравнения $n_{1} \cdots n_{k}=n$; $\tau_{2}(n)=\tau(n)$ - число делителей числа $n ; \pi(x)$ - количество простых чисел, не превосходящих $x ; \pi(x ; Q, l)$ - количество простых чисел, не превосходящих $x$ и лежащих в арифметической прогрессии вида $p \equiv l(\bmod q), 0<l<Q$, $(l, Q)=1 ; \chi(n), \chi_{1}(n), \chi_{2}(n)$ - неглавные характеры Дирихле по модулю $q$; $\chi_{0}(n)$ - главный характер Дирихле по модулю $q ;\left(\frac{n}{q}\right)$ - символ Лежандра; $g$ первообразный корень по модулю $q$; ind $n=\operatorname{ind}_{g} n$ - индекс числа $n$ по модулю $q$ при основании $g$; функции Чебышёва $\psi(x), \psi(x, \chi), \psi(x ; Q, l)$ определяются равенствами

$$
\begin{gathered}
\psi(x)=\sum_{n \leqslant x} \Lambda(n), \quad \psi(x, \chi)=\sum_{n \leqslant x} \Lambda(n) \chi(n), \\
\psi(x ; Q, l)=\sum_{\substack{n \leqslant x \\
n \equiv l(\bmod Q)}} \Lambda(n) ;
\end{gathered}
$$

запись $A \ll B$ означает неравенство $|A| \leqslant c B$, где $c>0, c$ - константа; то же самое означает запись $A=O(B) ; A \asymp B$ означает двустороннее неравенство $A \ll B \ll A$.

В каждой главе теоремы и леммы имеют свою нумерацию. 


\section{Глава I. Вспомогательные утверждения}

В этой главе сформулированы необходимые для дальнейшего вспомогательные утверждения - леммы.

\section{§1. Оценки полных сумм характеров}

Полной суммой будем называть сумму (11), в которой $X=q$.

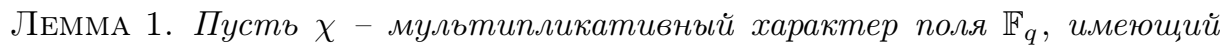
порядок $m>1$, и пусть $f \in \mathbb{F}_{q}[x]$ - нормированный многочлен положительной степени, не являющийся $m$-й степенъю другого многочлена. Если $d$ - число различных корней многочлена $f$ в его поле разложения над $\mathbb{F}_{q}$, то для каждого $a \in \mathbb{F}_{q}$ выполняется неравенство

$$
\left|\sum_{c \in \mathbb{F}_{q}} \chi(a f(c))\right| \leqslant(d-1) \sqrt{q} .
$$

Лемма 2. Пусть $1 \leqslant X<q, r$ - натуральное число. Тогда справедлива следующая оценка:

$$
\sum_{\lambda=1}^{q}\left|\sum_{x=1}^{X} \chi(\lambda+x)\right|^{2 r} \leqslant(4 r)^{r+1} X^{r} q+2 r X^{2 r} \sqrt{q} .
$$

Лемма 3. Пусть $a \not \equiv b(\bmod q), 1 \leqslant X<q, r-$ натуральное число. Тогда справедлива следующая оценка:

$$
\begin{aligned}
W & =\sum_{\lambda=1}^{q} \sum_{\mu=1}^{q}\left|\sum_{x=1}^{X} \chi_{1}(\lambda+\mu x+a) \chi_{2}(\lambda+\mu x+b)\right|^{2 r} \\
& \leqslant r^{2 r} X^{r} q^{2}+6 r^{2} X^{2 r} q .
\end{aligned}
$$

Лемма 1 - оценка Хассе-Вейля, ее доказательство см., например, в [6; с. 279]. Лемма 2 принадлежит Г. Дэвенпорту и П. Эрдёшу [42]. Лемма 3 для вещественного характера (символа Лежандра) доказана в [35; с. 316-317]. Для произвольных $\chi_{1}, \chi_{2}$ доказательство такое же, следует только в соответствующем месте рассуждений воспользоваться леммой 2.

\section{§ 2. Решето Виноградова}

Следующие три леммы, принадлежащие И. М. Виноградову, позволяют сводить сумму по простым числам к кратным суммам.

Лемма 4. Пусть $0<c \leqslant 1 / 6,0<\sigma \leqslant 1 / 3, \mathbb{P}$ - произведение простых чисел, не превосходящих $H=N^{\sigma}$. Тогда, полагая

$$
D=(\ln N)^{\frac{\ln \ln N}{\ln (1+c)}}
$$

делители $d$ чисел $\mathbb{P}$, не превосходящие $N$, можно распределить среди $K<D$ совокупностей $A_{1}, A_{2}, \ldots, A_{K}$ со следующими свойствами: 
1) числа $d$, принадлежащие одной и той же совокупности $A_{\nu}$, обладают одним и тем же числом $\beta$ простых сомножителей и, следовательно, одним и тем же значением $\mu(d)=(-1)^{\beta}$;

2) одна из совокупностей, пусть $A_{1}$, которую назовем простейшей, состоит из единственного числа $d=1 ;$ для этой совокупности положим $\varphi=1 u$, таким образом, будем иметь $d=\varphi=1$;

3) каждой из оставшихся совокупностей $A_{\nu}, \nu \geqslant 2$, отвечает свое $\varphi$ такое, что все числа $d$ этой совокупности удовлетворяют условию

$$
\varphi<d \leqslant \varphi^{1+c}
$$

4) для всякой совокупности $A_{\nu}, \nu \geqslant 2$, при любом $U$ с условием $0<U \leqslant \varphi$ существуют две совокупности $A_{\nu}^{\prime} u A_{\nu}^{\prime \prime}$ (вторая из них может оказатъся простейшей, т.е. $\left.A_{\nu}^{\prime \prime}=A_{1}\right)$ с отвечающими им значениями $\varphi^{\prime}$ и $\varphi^{\prime \prime}$ с условиями

$$
U<\varphi^{\prime} \leqslant U N^{\sigma}, \quad \varphi^{\prime} \varphi^{\prime \prime}=\varphi
$$

и такие, что при некотором целом положительном В каждое число из выбранной совокупности получим ровно $B$ раз, если из всех произведений $d^{\prime} d^{\prime \prime}$, $d^{\prime} \in A_{\nu}^{\prime}, d^{\prime \prime} \in A_{\nu}^{\prime \prime}$, выберем лишь удовлетворяющие условию $\left(d^{\prime}, d^{\prime \prime}\right)=1$.

Лемма 5. Пусть $\mathbb{P}$ - произведение всех простых чисел р с условием

$$
p \leqslant H \leqslant N^{\frac{1}{3}} .
$$

Определим натуральное число $k$ неравенствами

$$
H^{k} \leqslant N<H^{k+1} \text {. }
$$

Пусть $f(x)$ - комплекснозначная функиия с условием $|f(x)| \leqslant f_{1}$ и суммы $S$, $W_{\nu}, \nu=1, \ldots, k$, задаются равенствами

$$
\begin{gathered}
S=S(N)=\sum_{p \leqslant N} f(p), \\
W_{\nu}=\sum_{d_{1} \mid \mathbb{P}} \cdots \sum_{d_{\nu} \mid \mathbb{P}} \sum_{m_{1}>0} \cdots \sum_{m_{\nu}>0} \mu\left(d_{1}\right) \cdots \mu\left(d_{\nu}\right) f\left(d_{1} \cdots d_{\nu} m_{1} \cdots m_{\nu}\right) .
\end{gathered}
$$

Тогда для С справедлива следующая формула:

$$
S=\alpha_{1} W_{1}+\cdots+\alpha_{k} W_{k}+\theta \cdot 5 k \cdot 2^{k} f_{1} N H^{-1}(\ln N)^{k-1},
$$

¿де

$$
\left|\alpha_{\nu}\right| \leqslant 2^{k}, \quad \nu=1, \ldots, k ; \quad|\theta| \leqslant 1 .
$$

Лемма 6. Пусть $\mathbb{P}=\prod_{p \leqslant H} p, Q=\prod_{H<p \leqslant N} p, k$ - наибольшее иелое число с условием $H^{k} \leqslant N,|\theta(x)| \leqslant 1$,

$$
\begin{aligned}
W_{\nu} & =\sum_{d_{1} \mid \mathbb{P}} \cdots \sum_{\substack{d_{\nu} \mid \mathbb{P} \\
d_{1} \cdots d_{\nu} m_{1} \cdots m_{\nu} \leqslant N}} \cdots \sum_{m_{\nu}>0} \mu\left(d_{1}\right) \cdots \mu\left(d_{\nu}\right) \theta\left(d_{1} \cdots d_{\nu} m_{1} \cdots m_{\nu}\right), \\
W_{\nu}^{\prime} & =\sum_{\substack{y_{1} \mid Q \\
y_{1} \cdots y_{\nu} \leqslant N, \mu\left(y_{1} \cdots y_{\nu}\right)=0}} \cdots \sum_{\substack{y_{\nu} \mid Q \\
y_{1}}} \theta\left(y_{1} \cdots y_{\nu}\right), \quad S=\sum_{p \leqslant N} \theta(p) .
\end{aligned}
$$


Тогда при некоторых $\beta_{1}, \ldots, \beta_{k}, \beta_{1}^{\prime}, \ldots, \beta_{k}^{\prime}, c$, зависящих толъко от $k$, имеем равенство

$$
S=\beta_{1} W_{1}+\cdots+\beta_{k} W_{k}+\beta_{1}^{\prime} W_{1}^{\prime}+\cdots+\beta_{k}^{\prime} W_{k}^{\prime}+c H .
$$

Доказательства лемм 4, 5 и 6 см., например, в [50; с. 221 или с. 313], [37; леммы 10 и 11], а также в [51].

\section{Глава II. Линейные суммы характеров с простыми числами}

Здесь приводятся основные утверждения, относящиеся к оценкам линейных сумм характеров с простыми числами, и следствия этих оценок.

\section{§ 1. Суммы по подряд идущим простым числам}

Справедлива следующая

Теорема 1. Пусть $\omega-$ произвольное число из интервала $0<\omega<1 / 2$, $(a, q)=1, q^{0.5+\omega} \leqslant X \leqslant q^{2}$,

$$
S_{1}(X)=\sum_{p \leqslant X} \chi(p+a) .
$$

Тогда имеет место оценка:

$$
\left|S_{1}(X)\right| \ll X q^{-\frac{\omega^{2}}{1024}}
$$

где постоянная в знаке «зависит только от $\omega$.

При выполнении условий теоремы 1 справедливы следствия 1-3.

СледСтвиЕ 1 . Пусть $n \mid(q-1), 2 \leqslant n \leqslant q-2,0 \leqslant s \leqslant n-1$. Тогда для числа $T_{s}$ тех чисел $p+a$, которые удовлетворяют условиям

$$
p \leqslant X, \quad \operatorname{ind}(p+a) \equiv s(\bmod n),
$$

справедлива асимптотическая формула:

$$
T_{s}=\frac{1}{n} \pi(X)+O\left(X q^{-\frac{\omega^{2}}{1024}}\right) .
$$

СлЕДСТвИЕ 2. Число квадратичных вычетов и число квадратичных невычетов по модулю $q$ вида $p+a, p \leqslant X$, равно

$$
\frac{1}{2} \pi(X)+O\left(X q^{-\frac{\omega^{2}}{1024}}\right)
$$

СлЕДСТвиЕ 3. Число первообразных корней по модулю $q$ вида $p+a, p \leqslant X$, равно

$$
\frac{\varphi(q-1)}{q-1} \pi(X)+O\left(X q^{-\frac{\omega^{2}}{1025}}\right)
$$

Постоянные в знаках $O$ в следствиях 1-3 зависят только от $\omega$.

Доказательство теоремы 1 содержится в [33] (см. также [32]), доказательство следствий легко следует из теоремы 1 . Заметим также, что понижающий множитель в оценке теоремы 1 и в остаточных членах асимптотических формул следствий может быть заменен на $q^{-0.02 \omega^{2}}$. 


\section{§ 2. Суммы по простым числам, принадлежащим арифметической прогрессии}

Введем необходимые в этом параграфе обозначения: $A$ и $B$ - абсолютные постоянные, $A \geqslant 1, B \geqslant 1 ; Q$ - целое число, $(Q, q)=1,1 \leqslant Q \leqslant q^{A} ; l$ и $а$ целые положительные числа с условиями: $(l, Q)=(a, q)=1,0 \leqslant l \leqslant Q-1$, $1 \leqslant a \leqslant q-1$.

ТЕОРема 2. Пусть $\omega$ - произволъное число из интервала $\left(0, \frac{1}{2}\right)$ и Х удовлетворяет неравенствам

$$
Q^{3} q^{0.5+\omega} \leqslant X \leqslant Q^{3} q^{B}
$$

Тогда для сумми $S(X)$,

$$
S(X)=\sum_{\substack{p \leqslant X \\ p \equiv l(\bmod Q)}} \chi(p+a),
$$

справедливо неравенство

$$
|S(X)| \ll X Q^{-1} \Delta, \quad \Delta=q^{-0.0004 \omega^{2}},
$$

где постоянная в знаке «зависит только от $\omega$.

При выполнении условий теоремы 2 справедливы следствия 4-7.

СлЕДСТвИЕ 4. Пусть $V(X ; q, Q)$ u $N(X ; q, Q)$ - число квадратичных въчетов и число квадратичных невычетов по модулю $q$ вида $p+a, p \leqslant X$, $p \equiv l(\bmod Q)$. Тогда имеют место следующие асимптотические формуль:

$$
\begin{aligned}
V(X ; q, Q) & =\frac{1}{2} \pi(X ; Q, l)+O\left(X Q^{-1} q^{-0.0004 \omega^{2}}\right) ; \\
N(X ; q, Q) & =\frac{1}{2} \pi(X ; Q, l)+O\left(X Q^{-1} q^{-0.0004 \omega^{2}}\right) .
\end{aligned}
$$

СледСТвИЕ 5. Для любого є из интервала $(0,1)$ существует $\delta=\delta(\varepsilon)>0$ такое, что если

$$
q^{0.5+\varepsilon} \leqslant X \leqslant q^{2}
$$

u $Q$ - любое натуральное число, не превосходящее $q^{\delta}$, то при $q \geqslant q_{1}(\varepsilon)$ имеют место неравенства (обозначения следствия 1):

$$
\begin{aligned}
& V(X ; q, Q) \geqslant 0.4 X^{1-0.0001 \varepsilon^{2}} ; \\
& N(X ; q, Q) \geqslant 0.4 X^{1-0.0001 \varepsilon^{2}} .
\end{aligned}
$$

СледСтвие 6. Пусть $n-$ делитель $q-1,2 \leqslant n \leqslant q-2,0 \leqslant s \leqslant n-1$. Тогда для числа $T_{s}$ тех чисел $p+a$, которые удовлетворяют условиям

$$
p \leqslant X, \quad p \equiv l(\bmod Q), \quad \operatorname{ind}(p+a) \equiv s(\bmod n),
$$

справедлива формула:

$$
T_{s}=\frac{1}{n} \pi(X ; Q, l)+O\left(X Q^{-1} q^{-0,0004 \omega^{2}}\right) .
$$


СлеДСТВИЕ 7. Число первообразных корней по модулю $q$ вида $p+a, p \leqslant X$, $p \equiv l(\bmod Q)$, равно

$$
\frac{\varphi(q-1)}{q-1} \pi(X ; Q, l)+O\left(X Q^{-1} q^{-0.0003 \omega^{2}}\right) .
$$

Постоянные в знаках $O$ следствий 4, 6, 7 зависят только от $\omega$.

Доказательства следствий 4-7 легко следуют из теоремы 2, причем при доказательстве следствия 5 надо воспользоваться тЕоРемой Э. К. ФОГЕЛсА [52]: для любого $\varepsilon_{1}>0$ существует $A_{1}=A_{1}\left(\varepsilon_{1}\right)$ такое, что при $A \geqslant A_{1}$ имеет место неравенство:

$$
\pi\left(Q^{A} ; Q, l\right)>Q^{A\left(1-\varepsilon_{1}\right)}
$$

(теорема Фогелса обобщает известную теорему Ю. В. Линника [53] о наименьшем простом числе в арифметической прогрессии).

Теорема 2 доказана в [38]. Основой ее доказательства служат следующие ниже леммы 1-7, которые имеют также самостоятельный интерес, и леммы 4-6 главы I.

ЛЕмма 1. Пусть $r$ - натуральное число,

$$
W_{0}=\sum_{\lambda=0}^{q-1}\left|\sum_{m=1}^{M_{0}} \psi_{0}(m) \chi(m+\lambda)\right|^{2 r},
$$

$\psi_{0}(m)$ - произвольная комплекснозначная функиия $m$ u $\left|\psi_{0}(m)\right| \leqslant H_{0}$ при $1 \leqslant$ $m \leqslant M_{0}$. Тогда для величины $W_{0}$ справедлива следующая оченка:

$$
W_{0} \ll H_{0}^{2 r} q M_{0}^{2 r} \Delta_{0}, \quad \Delta_{0}=q^{-0.5}+M_{0}^{-r},
$$

причем постоянная в знаке «зависит только от $r$.

ЛЕмма 2. Пусть $r$ - натуральное число,

$$
W=\sum_{\lambda=0}^{q-1}\left|\sum_{\substack{m=1 \\ m \equiv l(\bmod Q)}}^{M} \psi(m) \chi(m+\lambda)\right|^{2 r},
$$

$\psi(m)$ - произвольная комплекснозначная функция $m u|\psi(m)| \leqslant H$ при $1 \leqslant$ $m \leqslant M$. Тогда для $W$ справедлива следующая оченка:

$$
W \ll H^{2 r}\left(M Q^{-1}+1\right)^{2 r} q \Delta, \quad \Delta=q^{-0.5}+\left(M Q^{-1}+1\right)^{-r},
$$

причем постоянная в знаке «зависит только от $r$.

Лемма 3. Пусть $\gamma>0, q^{\frac{1}{4}+\gamma} \leqslant M \leqslant q^{\frac{5}{8}}, 0 \leqslant a \leqslant q-1 u$

$$
T(M)=\sum_{m=1}^{M} \chi(m+a) .
$$

Тогда для $|T(M)|$ имеет место следующая оценка:

$$
|T(M)| \ll M q^{\varepsilon} \Delta, \quad \Delta=q^{-\frac{1}{2} \gamma^{2}},
$$

где постоянная в знаке «зависит только от є $и \gamma$. 
Лемма 4. Пусть $0<\gamma<\frac{1}{2}, M Q^{-1} \geqslant q^{0.25+\gamma}, 0 \leqslant a \leqslant q-1 u$

$$
T=\left|\sum_{\substack{m=1 \\ m \equiv l(\bmod Q)}}^{M} \chi(m+a)\right| .
$$

Тогда для Т справедлива следующая ощенка:

$$
T \ll M Q^{-1} q^{\varepsilon} \Delta, \quad \Delta=q^{-0.5 \gamma^{2}},
$$

причем постоянная в знаке «зависит только от $\gamma$.

Лемма 5. Рассмотрим сумму $W_{\alpha}$,

$$
W_{\alpha}=\sum_{\substack{U<u \leqslant U_{1} \\ m u \leqslant N, m u \equiv l(\bmod Q)}} \sum_{\substack{M<m \leqslant M_{1} \\ m u \leqslant}} \psi_{1}(u) \psi_{2}(m) \chi(m u+k),
$$

где $U<U_{1} \leqslant 2 U, M<M_{1} \leqslant 2 M,\left|\psi_{1}(u)\right| \leqslant H_{1},\left|\psi_{2}(m)\right| \leqslant H_{2}$. Тогда если $(k, q)=1 u$

$$
U Q^{-1} \geqslant q^{0.5+\gamma_{1}}, \quad M Q^{-1} \geqslant q^{\gamma}
$$

при некоторых положительных $\gamma_{1}$ и $\gamma$, не превосходящих 0.5, то для $\left|W_{\alpha}\right|$ имеет место оценка

$$
\left|W_{\alpha}\right| \ll H_{1} H_{2} U M Q^{-1} \Delta, \quad \Delta=q^{-0.25 \gamma \gamma_{1}},
$$

причем постоянная в знаке «зависит только от $\gamma$.

Лемма 6. Рассмотрим сравнение

$$
m_{1}-m_{2} \equiv k_{0}\left(u_{2}^{-1}-u_{1}^{-1}\right)(\bmod q),
$$

где $\left(k_{0}, q\right)=1$ и переменные $m_{1}, m_{2}, u_{1}, u_{2}$ удовлетворяют условиям: $1 \leqslant$ $M<m_{1}, m_{2} \leqslant 2 M, U<u_{1}, u_{2} \leqslant 2 U, u_{1} m_{1} \equiv u_{2} m_{2} \equiv l(\bmod Q),(l, Q)=1$, $0 \leqslant l<Q, Q \leqslant M \leqslant U$. Для числа $j$ решений этого сравнения справедлива следующая оценка:

$$
j \ll q^{\varepsilon} M U Q^{-1} \Delta_{0},
$$

¿əe

$$
\Delta_{0}=Q+q^{-0.5} U^{0.5} M^{1.5} Q^{-1}+q^{-1} U M^{2} Q^{-1},
$$

а постоянная в знаке « зависит только от $\varepsilon$.

Лемма 7. Рассмотрим сумму $W_{\beta}$,

$$
W_{\beta}=\sum_{\substack{U<u \leqslant 2 U \\ m u \leqslant N, m u \equiv l(\bmod Q)}} \sum_{\substack{M<m \leqslant M_{1} \\ m u}} \psi(u) \chi(m u+k),
$$

где $(k, q)=1, M<M_{1} \leqslant 2 M,|\psi(u)| \leqslant H$. Предположим, далее, что величины $U, M$ и $Q$ удовлетворяют следующим соотношениям:

$$
M Q^{-1} \geqslant q^{\gamma}, \quad M U Q^{-2} \geqslant q^{0.5+\gamma_{1}}, \quad M \leqslant q^{0.5-\gamma_{2}}, \quad U M^{-1} \geqslant q^{\gamma_{3}}
$$


с некоторыми положительными $\gamma, \gamma_{1}, \gamma_{2}, \gamma_{3}$, причем $\gamma<0.333$. Тогда

$$
\left|W_{\beta}\right| \ll q^{\varepsilon} H U M Q^{-1} \Delta,
$$

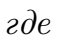

$$
\Delta=q^{-0.125 \gamma}+q^{-0.5 \gamma \gamma_{1}}+q^{-0.5 \gamma \gamma_{2}}+q^{-0.5 \gamma \gamma_{3}},
$$

а постоянная в знаке «зависит только от $\gamma u \varepsilon$.

ЗАмЕчАниЕ. Понижающий множитель $\Delta$ в оценке теоремы 2 и в остаточных членах асимптотических формул следствий 4-7 может быть заменен на $q^{-0.02 \omega^{2}}$.

\section{§ 3. Распределение произведений сдвинутых простых чисел в коротких арифметических прогрессиях}

В этом параграфе решается задача, связанная с распределением чисел вида $p\left(p^{\prime}+a\right)$, где $p, p^{\prime}$ - простые числа, в арифметических прогрессиях с растущей разностью $q$ (см. [54]). Метод решения является мультипликативным аналогом метода Виноградова решения тернарных аддитивных проблем теории чисел. Как это было отмечено в [54], этим методом можно решать, в частности, задачи о распределении простых чисел в арифметических прогрессиях "в среднем".

Прежде чем сформулировать теорему, введем необходимые новые обозначения. Пусть $\omega \in(0,1 / 4], n$ - достаточно большое положительное число, т. е. $n \geqslant n^{\prime}(\omega)$; пусть далее $\varkappa_{0} \cdot(4.6+\omega)=1, q \leqslant n^{\varkappa_{0}} ;(a, q)=(l, q)=1 ; \alpha-$ произвольное число из отрезка

$$
\left[\left(\frac{1}{2}+\omega\right) \frac{\ln q}{\ln n}, 1-4.1 \frac{\ln q}{\ln n}\right] ;
$$

$n_{1} \geqslant n^{1-\alpha}, n_{2} \geqslant n^{\alpha} ; p, p^{\prime}-$ простые числа; $\pi_{2}=\pi_{2}\left(n_{1}, n_{2} ; a, l\right)-$ количество чисел $p \leqslant n_{1}, p^{\prime} \leqslant n_{2}$ таких, что $p\left(p^{\prime}+a\right) \equiv l(\bmod q)$.

ТЕОРема 3. Существует абсолютная постоянная $\gamma>0$ такая, что

$$
\pi_{2}=\frac{1}{q-1} \pi\left(n_{1}\right) \pi\left(n_{2}\right)+O\left(\left(n_{1} n_{2}\right)^{1+\varepsilon} q^{-1-\gamma \omega^{2}}\right),
$$

где постоянная в знаке $О$ зависит только от $\omega$ и $\varepsilon$.

При доказательстве теоремы будем пользоваться следующей леммой.

Лемма 8. Пусть $\psi_{1}(u)$ и $\psi_{2}(v)$ - произвольные комплекснозначные функuиu, $\left|\psi_{1}(u)\right| \ll u^{\varepsilon},\left|\psi_{2}(v)\right| \ll v^{\varepsilon}$. Пусть, далее,

$$
\begin{gathered}
q \ll U<U_{1} \leqslant 2 U, \quad q \ll V<V_{1} \leqslant 2 V \\
W=\frac{1}{q-1} \sum_{\chi \bmod q}\left|\sum_{\substack{U<u \leqslant U_{1} \\
u v \leqslant X}} \sum_{\substack{V<v \leqslant V_{1} \\
u}} \psi_{1}(u) \psi_{2}(v) \chi(u v)\right| .
\end{gathered}
$$

Тогда

$$
W \ll(U V)^{1+\varepsilon} q^{-1} \ll X^{1+\varepsilon} q^{-1}
$$


ДокАЗАТЕЛЬСТво тЕОРЕМЫ 3. Имеем равенство:

$$
\begin{aligned}
\pi_{2} & =\frac{1}{\varphi(q)} \sum_{\chi \bmod q} \sum_{p \leqslant n_{1}} \sum_{p^{\prime} \leqslant n_{2}} \chi\left(p\left(p^{\prime}+a\right)\right) \overline{\chi(l)} \\
& =\frac{1}{\varphi(q)} \pi\left(n_{1}\right) \pi\left(n_{2}\right)+R+O\left(n_{1} n_{2} q^{-2}\right)
\end{aligned}
$$

где

$$
|R| \leqslant \frac{1}{\varphi(q)} \sum_{\chi \neq \chi_{0}}\left|\sum_{p \leqslant n_{1}} \chi(p)\right|\left|\sum_{p \leqslant n_{2}} \chi(p+a)\right| .
$$

Пользуясь теоремой 1 , получаем:

$$
|R| \leqslant n_{2} q^{-\gamma \omega^{2}} R_{1}
$$

где

$$
R_{1}=\frac{1}{\varphi(q)} \sum_{\chi \neq \chi_{0}}\left|\sum_{p \leqslant n_{1}} \chi(p)\right|
$$

Обозначая $N=n_{1}$, возьмем в лемме 6 главы I $H=\max \left(N^{0.1}, \sqrt{q}\right)$ и применим ее к внутренней сумме $R_{1}$. Получим неравенство

$$
R_{1} \ll \sum_{1 \leqslant s \leqslant s_{0}} \frac{1}{\varphi(q)} \sum_{\chi \neq \chi_{0}}\left(\left|W_{s}\right|+\left|W_{s}^{\prime}\right|\right)+H,
$$

причем $s_{0} \leqslant 10$, а константа в знаке « абсолютная. Из определения сумм $W_{s}^{\prime}$ следует:

$$
\frac{1}{\varphi(q)} \sum_{\chi \neq \chi_{0}}\left|W_{s}^{\prime}\right| \ll N^{\varepsilon} \sum_{H<d \leqslant N} \sqrt{\frac{N}{d^{2}}\left(\frac{N}{q d^{2}}+1\right)} \ll N^{1+\varepsilon} q^{-1} .
$$

Осталось оценить при каждом $s, 1 \leqslant s \leqslant s_{0}$, суммы $R_{2}$,

$$
R_{2}=\frac{1}{\varphi(q)} \sum_{\chi \neq \chi_{0}}\left|W_{s}\right|
$$

Возьмем в лемме 4 главы I число $c=1 / 60$. Все делители $d \mid \mathbb{P}, d \leqslant N$, распределим среди $D_{1} \leqslant(\ln N)^{(\ln \ln N) / \ln (1+c)}$ совокупностей. Кроме того, интервалы $0<m_{i} \leqslant N, 1 \leqslant i \leqslant s$, разобьем на $\ll \ln N$ интервалов вида $M_{i}<m_{i} \leqslant M_{i}^{\prime} \leqslant 2 M_{i}$. Получим $\ll Q_{1}(\ln N)^{s}$ сумм $R_{3}$ вида

$$
R_{3}=\frac{1}{\varphi(q)} \sum_{\chi \neq \chi_{0}}\left|\sum_{d_{1}} \cdots \sum_{m_{s}} \mu\left(d_{1}\right) \cdots \mu\left(d_{s}\right) \chi\left(d_{1} \cdots d_{s} m_{1} \cdots m_{s}\right)\right|
$$

где суммирование ведется по области $M_{i}<m_{i} \leqslant M_{i}^{\prime}, \varphi_{i}<d_{i} \leqslant \varphi_{i}^{1+c}, i=$ $1, \ldots, s, m_{1} \cdots m_{s} d_{1} \cdots d_{s} \leqslant N$. 
Достаточно рассмотреть случай $M_{1} \cdots M_{s}\left(\varphi_{1} \cdots \varphi_{s}\right)^{1+c} \geqslant N q^{-\frac{1}{2}}$, так как в противном случае тривиально имеем $R_{3} \ll N^{1+\varepsilon} q^{-1}$.

Обозначим произведение $M_{1} \cdots M_{s} \varphi_{1} \cdots \varphi_{s}$ буквой $\Phi$. Тогда: или а) $\Phi=$ $\Phi_{1} \Phi_{2}$, где $\Phi_{1} \geqslant q, \Phi_{2} \geqslant q, \Phi_{1}=M_{i_{1}} \cdots M_{i_{r}} \varphi_{j_{1}} \cdots \varphi_{j_{k}}$, или б) представление а) невозможно.

Случай а). Полагая

$$
u=m_{i_{1}} \cdots m_{i_{r}} d_{j_{1}} \cdots d_{j_{k}}, \quad v=d_{1} \cdots d_{s} m_{1} \cdots m_{s} u^{-1},
$$

получим:

$$
R_{3}=\frac{1}{\varphi(q)} \sum_{\chi \neq \chi_{0}}\left|\sum_{\substack{U<u \leqslant U_{1} \\ u v \leqslant N}} \sum_{\substack{V<v \leqslant V_{1} \\ \psi_{1}}} \psi_{1}(u) \psi_{2}(v) \chi(u v)\right|
$$

Разбивая интервалы изменения $u$ и $v$ на $\ll \ln N$ интервалов вида

$$
U_{2}<u \leqslant U_{2}^{\prime} \leqslant 2 U_{2}, \quad V_{2}<v \leqslant V_{2}^{\prime} \leqslant 2 V_{2}
$$

и замечая, что $U_{2} \geqslant q, V_{2} \geqslant q$, получим $\ll \ln ^{2} N$ сумм $R_{3}^{\prime}$, к каждой из которых применима оценка леммы 8. Таким образом,

$$
R_{3} \ll N^{1+\varepsilon} q^{-1}
$$

Случай б). Здесь возможны два подслучая:

$$
\text { 1) } \max _{1 \leqslant i \leqslant s} M_{i} \geqslant \Phi q^{-1} ; \quad \text { 2) } \max _{1 \leqslant i \leqslant s} \varphi_{i} \geqslant \Phi q^{-1} \text {. }
$$

Подслучай 1). Пусть $\max _{1 \leqslant i \leqslant s} M_{i}=M_{1}, u=m_{1}, v=m_{2} \cdots m_{s} d_{1} \cdots d_{s}$. Тогда

$$
\begin{gathered}
\Phi q^{-1} \leqslant M_{1}<u \leqslant M_{1}^{\prime}, \quad u v=m_{1} \cdots m_{s} d_{1} \cdots d_{s} \leqslant \Phi\left(\varphi_{1} \cdots \varphi_{s}\right)^{c} \\
\varphi_{1} \cdots \varphi_{s} \leqslant q, \quad v \leqslant q^{1+c} .
\end{gathered}
$$

Поэтому $R_{3}$ не превосходит $\ll \ln N$ сумм $R_{3}^{\prime}$ вида

$$
R_{3}^{\prime}=\frac{1}{\varphi(q)} \sum_{\chi \neq \chi_{0}}\left|\sum_{V_{2}<v \leqslant V_{2}^{\prime}} \psi_{2}(v) \chi(v) \sum_{M_{1}<u \leqslant M_{1}^{\prime \prime}} \chi(u)\right|,
$$

где $M_{1}^{\prime \prime}=\min \left(M_{1}^{\prime}, N v^{-1}\right)$. Следовательно,

$$
R_{3}^{\prime} \ll q^{1+c} \sqrt{q} \ln q \ll N^{1+\varepsilon} q^{-1}, \quad R_{3} \ll N^{1+\varepsilon} q^{-1} .
$$

Подслучай 2). Пусть $\max _{1 \leqslant i \leqslant s} \varphi_{i}=\varphi_{j}=\varphi$. Тогда

$$
\varphi \geqslant \Phi q^{-1} \geqslant N^{\frac{1}{1+c}} q^{-1-\frac{1}{2(1+c)}}
$$


В лемме 4 гл. I положим $U=q^{\frac{3}{2}}$. Последовательно получаем: $U<\varphi^{\prime} \leqslant U H$, $\varphi^{\prime} \varphi^{\prime \prime}=\varphi$,

$$
\begin{aligned}
R_{3}= & \frac{1}{\varphi(q)} \sum_{\chi \neq \chi_{0}} \mid \sum_{\varphi^{\prime}<d^{\prime} \leqslant\left(\varphi^{\prime}\right)^{1+c}} \mu\left(d^{\prime}\right) \sum_{\substack{\varphi^{\prime \prime}<d^{\prime \prime} \leqslant\left(\varphi^{\prime \prime}\right)^{1+c} \\
\left(d^{\prime}, d^{\prime \prime}\right)=1}} \mu\left(d^{\prime \prime}\right) \\
& \ldots \sum_{M_{s}<m_{s} \leqslant M_{s}^{\prime}} \chi\left(d^{\prime} d^{\prime \prime} \cdots m_{s}\right) \mid \\
= & \frac{1}{\varphi(q)} \sum_{\chi \neq \chi_{0}} \mid \sum_{d \leqslant N} \mu(d) \chi\left(d^{2}\right) \sum_{\varphi^{\prime} d^{-1}<d^{\prime} \leqslant\left(\varphi^{\prime}\right)^{1+c} d^{-1}} \mu\left(d d^{\prime}\right) \chi\left(d^{\prime}\right) \\
& \times \frac{1}{\varphi(q)} \sum_{\chi \neq \chi_{0}}\left|\sum_{d \leqslant \sqrt{q}} K(d)\right|+\frac{1}{\varphi(q)} \sum_{\chi \neq \chi_{0}}\left|\sum_{d>\sqrt{q}} K(d)\right|,
\end{aligned}
$$

где

$$
K(d)=\mu(d) \chi\left(d^{2}\right) \sum_{d^{\prime}} \sum_{\substack{d^{\prime \prime} \\ d^{\prime} d^{\prime \prime} \cdots m_{s} \leqslant N d^{-2}}} \cdots \sum_{\substack{M_{s}<m_{s} \\ \text { s. }}} \mu\left(d d^{\prime}\right) \chi\left(d^{\prime}\right) \mu\left(d d^{\prime \prime}\right) \chi\left(d^{\prime \prime} \cdots m_{s}\right) .
$$

Для второй суммы в правой части неравенства (21) имеем оценку:

$$
\begin{aligned}
& \ll \sum_{\sqrt{q}<d \leqslant N} \frac{1}{\varphi(q)} \sum_{\chi \neq \chi_{0}}|K(d)|=\sum_{\sqrt{q}<d \leqslant N} \frac{1}{\varphi(q)} \sum_{\chi \neq \chi_{0}}\left|\sum_{u \leqslant N d^{-2}} \psi_{1}(u) \chi(u)\right| \\
& \ll N^{\varepsilon} \sum_{\sqrt{q}<d \leqslant N} \sqrt{\frac{N}{d^{2}}\left(\frac{N}{q d^{2}}+1\right)} \ll N^{1+\varepsilon} q^{-1} .
\end{aligned}
$$

Рассмотрим теперь первую сумму в правой части (21). Слагаемые в $K(d)$ имеют вид $\psi_{1}(u) \psi_{2}(v) \chi(u) \chi(v)$, где $u=d^{\prime}, v=d^{\prime \prime} \cdots m_{s}$. Разбивая интервалы изменения величин $u$ и $v$ на интервалы подобно тому, как это делалось выше, получим $\ll \ln ^{2} N$ сумм вида

$$
\frac{1}{\varphi(q)} \sum_{\chi \neq \chi_{0}}\left|\sum_{\substack{U<u \leqslant U_{1} \\ u v \leqslant N d^{-2}}} \sum_{\substack{V<v \leqslant V_{1} \\ \psi_{1}}} \psi_{1}(u) \psi_{2}(v) \chi(u v)\right|
$$

причем $U \gg \varphi^{\prime} q^{-\frac{1}{2}} \geqslant q, V \gg \varphi^{\prime \prime} q^{-\frac{1}{2}} \cdots M_{s}=q^{-\frac{1}{2}} \Phi\left(\varphi^{\prime}\right)^{-1-c} \gg q$. Применяя к (22) лемму 8, получим:

$$
|K(d)| \ll N^{1+\varepsilon} q^{-1} d^{-2}, \quad \sum_{d \leqslant \sqrt{q}}|K(d)| \ll N^{1+\varepsilon} q^{-1} .
$$

Следовательно,

$$
R_{3} \ll N^{1+\varepsilon} q^{-1}
$$


Итак, для $R_{1}$ получили оценку

$$
R_{1} \ll N^{1+\varepsilon} q^{-1}
$$

Из этой оценки и (20) следует утверждение теоремы 3.

\section{Глава III. Нелинейные суммы характеров с простыми числами}

Здесь приводятся основные утверждения, относящиеся к оценкам нелинейных сумм характеров с простыми числами, и следствия этих оценок. В этой главе $a$ и $b$ - целые числа, $a \not \equiv b(\bmod q)$, в следствиях $2,4,5,7$, кроме того, $a b \not \equiv 0(\bmod q)$.

\section{§ 1. Суммы по подряд идущим простым числам}

Справедлива следующая

ТеОрема 1. Пусть $\omega$ - произвольное число из интервала $\left(0, \frac{1}{2}\right), f(x)=$ $(x+a)(x+b)$. Тогда при любом $X \geqslant q^{0.75+\omega}$ для суммы $S_{2}(X)$,

$$
S_{2}(X)=\sum_{p \leqslant X} \chi(f(p)),
$$

справедлива оченка

$$
\left|S_{2}(X)\right| \ll \pi(X) q^{-0.02 \omega^{2}}
$$

где постоянная в знаке «зависит только от $\omega$.

При выполнении условий теоремы 1 справедливы следствия 1-4.

СлЕДСТвИЕ 1. Число квадратичных вычетов (невычетов) по модулю $q$ в последовательности вида $f(p), p \leqslant X$, равно

$$
\frac{1}{2} \pi(X)+O\left(\pi(X) q^{-0.02 \omega^{2}}\right)
$$

СлеДСтвиЕ 2. Пусть $s_{1}= \pm 1, s_{2}= \pm 1$. Тогда число простых чисел $p$, не превосходящих $X$ и таких, что

$$
\left(\frac{p+a}{q}\right)=s_{1}, \quad\left(\frac{p+b}{q}\right)=s_{2},
$$

равно

$$
T_{1}=T_{1}(X)=\frac{1}{4} \pi(X)+O\left(\pi(X) q^{-0.02 \omega^{2}}\right) .
$$

СлЕДСтвиЕ 3. Число первообразнъх корней в последователъности $f(p)$, $p \leqslant X$, равно

$$
\frac{\varphi(q-1)}{q-1} \pi(X)+O\left(\pi(X) q^{-0.01 \omega^{2}}\right)
$$


СЛЕДСТВИЕ 4. Число простых чисел $p \leqslant X$, для которых числа $p+a u p+b$ одновременно являются первообразными корнями по модулю q, равно

$$
T_{2}=T_{2}(X)=\left(\frac{\varphi(q-1)}{q-1}\right)^{2} \pi(X)+O\left(\pi(X) q^{-0.01 \omega^{2}}\right) .
$$

В следствиях 1-4 постоянные в знаках $O$ зависят только от $\omega$.

Доказательство теоремы 1 с несколько худшим понижающим множителем для $\chi(n)=\left(\frac{n}{q}\right)$ содержится в [35]. Случай произвольного характера $\chi(n)$ рассматривается так же, только в нужном месте следует воспользоваться леммой 3 главы I с $\chi_{1}=\chi_{2}=\chi$. Доказательства следствий легко следуют из теоремы 1.

\section{§ 2. Суммы по простым числам, принадлежащим арифметической прогрессии}

В этом параграфе считаем, что $q \geqslant q_{0} ; Q$ - натуральное число, $(Q, q)=1$, $1 \leqslant Q \leqslant q^{A} ; l$ - натуральное число, $(l, Q)=1,1 \leqslant l<Q ; A$ и $B$ - произвольные абсолютные постоянные, большие $1 ; f(x)=(x+a)(x+b)$.

Теорема 2. Пусть Х удовлетворяет неравенствам

$$
Q^{3} q^{0.75+\omega} \leqslant X \leqslant Q^{3} q^{B}, \quad 0<\omega<\frac{1}{2} .
$$

Тогда для суммъ $S_{3}(X)$,

$$
S_{3}(X)=\sum_{\substack{p \leqslant X \\ p \equiv l(\bmod Q)}} \chi(f(p))
$$

справедлива следующая оценка:

$$
\left|S_{3}(X)\right| \ll Q^{-1} X q^{-0.02 \omega^{2}},
$$

где постоянная в знаке «зависит только от $\omega$.

При выполнении условий теоремы 2 справедливы следствия 5-8.

СлЕДСТвИЕ 5. Пусть $s_{1}= \pm 1, s_{2}= \pm 1, T_{1}(X)$ - число простых чисел $p \leqslant X$ maкux, чmо

$$
p \equiv l(\bmod Q), \quad\left(\frac{p+a}{q}\right)=s_{1}, \quad\left(\frac{p+b}{q}\right)=s_{2} .
$$

Тогда

$$
T_{1}(X)=\frac{1}{4} \pi(X ; Q, l)+O\left(Q^{-1} X q^{-0.02 \omega^{2}}\right) .
$$

СлеДСтвиЕ 6. Пусть $T_{2}(X)$ - число простых чисел $p \leqslant X$ maких, что $p \equiv l(\bmod Q) u(p+a)(p+b)-$ первообразный корень по модулю $q$. Тогда

$$
T_{2}(X)=\frac{\varphi(q-1)}{q-1} \pi(X ; Q, l)+O\left(Q^{-1} X q^{-0.01 \omega^{2}}\right) .
$$


СЛЕДСТвИЕ 7. Пусть $T_{3}(X)$ - число простых чисел $p \leqslant X$ maких, что $p \equiv l(\bmod Q) u p+a, p+b$ одновременно являются первообразными корнями по модулю $q$. Тогда

$$
T_{3}(X)=\left(\frac{\varphi(q-1)}{q-1}\right)^{2} \pi(X ; Q, l)+O\left(Q^{-1} X q^{-0.01 \omega^{2}}\right) .
$$

СлеДСТВИЕ 8. Для любого в из интервала $(0,1)$ существует $\delta=\delta(\varepsilon)>0$ такое, что если

$$
q^{0.75+\varepsilon} \leqslant X \leqslant q^{2}
$$

и $Q$ - любое натуральное число, не превосходящее $q^{\delta}$, то при $q \geqslant q_{1}(\varepsilon) u$ обозначениях следствий 5-7 имеют место неравенства:

$$
T_{\nu}(X) \geqslant X^{1-0.01 \varepsilon^{2}}, \quad \nu=1,2,3 .
$$

Постоянные в знаках $O$ следствий 5-7 зависят только от $\omega$.

Доказательство теоремы 2 с несколько худшим понижающим множителем для $\chi(n)=\left(\frac{n}{q}\right)$ содержится в [21].

Основу доказательства теоремы 2 составляют следующие две леммы, которые имеют также самостоятельный интерес. Эти леммы являются нелинейными аналогами лемм 7 и 5 главы II.

Лемма 1. Пусть числа $M$ и U удовлетворяют неравенствам:

$$
\begin{gathered}
Q^{2} q^{0.75+\omega} \leqslant M U \leqslant Q^{2} q^{2}, \quad M>Q q^{\omega}, \\
M<M_{1} \leqslant 2 M, \quad U<U_{1} \leqslant 2 U,
\end{gathered}
$$

и комплекснозначная функция $\psi(u)$ на отрезке $\left[U, U_{1}\right]$ по абсолютной величине не превосходит $\psi_{0}>0$. Тогда для двойной суммъ $W_{1}$,

$$
W_{1}=\sum_{\substack{U<u \leqslant U_{1} \\ m u \leqslant N, m u \equiv l(\bmod Q)}} \sum_{\substack{M<m \leqslant M_{1} \\ m u \leqslant}} \psi(u) \chi((m u+a)(m u+b))
$$

справедлива оченка

$$
\left|W_{1}\right| \leqslant c_{1}(\omega) \psi_{0} Q^{-1} M U q^{-0.05 \omega^{2}}
$$

ДокАЗАтельство. Возможны следующие три случая для значений величин $M$ и $U$, каждый из которых будет рассмотрен отдельно: 1$) M \geqslant Q q^{0.5+0.05 \omega}$; 2) $M<Q q^{0.5+0.05 \omega}, U>Q q^{0.5+0.05 \omega}$; 3) $M<Q q^{0.5+0.05 \omega}, U<q^{0.5+0.05 \omega}$.

Cлучай 1). По условию леммы $(l, Q)=1$, поэтому $(u, Q)=(m, Q)=1$. Количество чисел $u$, кратных $q$, не более $U q^{-1}+1$. Поэтому, выделяя в $W_{1}$ те слагаемые, для которых $u$ кратны $q$, и пользуясь тем, что $m \equiv l u^{-1}(\bmod Q)$, $|\psi(u)| \leqslant \psi_{0}, M<m \leqslant M_{1}$, находим тривиальную оценку этой части суммы $W_{1}$ :

$$
\ll\left(U q^{-1}+1\right) M Q^{-1} \psi_{0} \ll \psi_{0} Q^{-1} M U q^{-1} .
$$

Заметим, что здесь мы воспользовались соотношением $U \gg q$, которое тривиально выполняется, так как предполагается существование $u \equiv 0(\bmod q)$, 
$U<u \leqslant U_{1} \leqslant 2 U$. Пусть теперь $W_{2}$ означает часть суммы $W_{1}$ по числам $u$ с условием $(u, q)=1$. Легко находим

$$
W_{2} \ll \sum_{U<u \leqslant U_{1}} \psi_{0}\left|\sum_{\substack{M<m \leqslant M_{2} \\ m \equiv l_{1}(\bmod Q)}} \chi\left(\left(m+a_{1}\right)\left(m+b_{1}\right)\right)\right|,
$$

где $u l_{1} \equiv l(\bmod Q), u a_{1} \equiv a(\bmod q), u b_{1} \equiv b(\bmod q), M_{2}=\min \left(M_{1}, N u^{-1}\right)$. Представим $m$ в виде

$$
m=Q r+l_{1}, \quad R=\left(M-l_{1}\right) Q^{-1}<r \leqslant\left(M_{2}-l_{1}\right) Q^{-1}=R_{1} .
$$

Тогда внутренняя сумма перепишется так:

$$
\sum_{R<r \leqslant R_{1}} \chi\left(\left(Q r+l_{1}+a_{1}\right)\left(Q r+l_{1}+b_{1}\right)\right)=\chi\left(Q^{2}\right) \sum_{R<r \leqslant R_{1}} \chi\left(\left(r+k_{1}\right)\left(r+k_{2}\right)\right),
$$

где $Q k_{1} \equiv l_{1}+a_{1}(\bmod q), Q k_{2} \equiv l_{1}+b_{1}(\bmod q)$. Так как $k_{1} \not \equiv k_{2}(\bmod q)$, то для последней суммы справедлива оценка:

$$
\ll\left(\left(R_{1}-R\right) q^{-1}+1\right) \sqrt{q} \ln q .
$$

Вспоминая, что $M \geqslant Q q^{0.05 \omega+0.5}$, для $W_{2}$ получаем неравенство:

$$
\begin{aligned}
W_{2} & \ll U \psi_{0}\left(M Q^{-1} q^{-1}+1\right) \sqrt{q} \ln q \\
& =\psi_{0} M U\left(Q^{-1} q^{-1}+M^{-1}\right) \sqrt{q} \ln q \ll \psi_{0} Q^{-1} M U q^{-0.05 \omega} \ln q .
\end{aligned}
$$

Отсюда и из ранее полученной оценки части суммы $W_{1}$ следует, что в случае 1 ) утверждение леммы справедливо.

Случай 2). Переходя к неравенствам, будем иметь:

$$
\left|W_{1}\right| \leqslant \sum_{U<u \leqslant U_{1}} \psi_{0}\left|\sum_{\substack{M<m \leqslant M_{2} \\ m \equiv l_{1}(\bmod Q)}} \chi((m u+a)(m u+b))\right| .
$$

Как и в первом случае, представим $m$ и $u$ в виде

$$
m=Q r+l_{1}, \quad u=Q v+u_{1},
$$

где

$$
\begin{gathered}
R=\left(M-l_{1}\right) Q^{-1}<r \leqslant\left(M_{2}-l_{1}\right) Q^{-1}=R_{2}, \quad 0<u_{1}<Q, \\
\left(u_{1}, Q\right)=1, \quad V=\left(U-u_{1}\right) Q^{-1}<v \leqslant\left(U_{1}-u_{1}\right) Q^{-1}=V_{1} .
\end{gathered}
$$

Легко находим:

$$
\left|W_{1}\right| \leqslant \psi_{0} Q \max _{u_{1}} \sum_{V<v \leqslant V_{1}}\left|\sum_{R<r \leqslant R_{2}} \chi\left(f\left(Q r+l_{1}\right)\left(Q v+u_{1}\right)\right)\right|,
$$

причем $l_{1}$ определяется сравнением $l_{1} u_{1} \equiv l(\bmod Q)$ и, следовательно, от $v$ не зависит; от $v$ зависит $R_{2}$. Так как $R<q^{0.5+0.05 \omega}$, то возможно только одно 
значение для $r$, при котором $Q r+l_{1} \equiv 0(\bmod q)$. Исключая его, если оно есть, получим погрешность $\ll \psi_{0} Q V$. Возведем далее последнее неравенство в квадрат и воспользуемся неравенством Коши. Применяя очевидные сокращенные обозначения, найдем:

$$
\left|W_{1}\right|^{2} \ll \psi_{0}^{2} Q^{2} V \sum_{V<v \leqslant V_{1}}\left|\sum_{R<r \leqslant R_{2}}\right|^{2} \ll \psi_{0}^{2} Q^{2} V \sum_{R<r_{1}, r_{2} \leqslant 2 R}\left|\sum_{V<v \leqslant V_{2}}\right| .
$$

Сумму по $r_{1}, r_{2}$ разобьем на две части: в одну сумму объединим слагаемые с условием $r_{1}=r_{2}$ или $a\left(Q r_{1}+l_{1}\right)^{-1} \equiv b\left(Q r_{2}+l_{1}\right)^{-1}(\bmod q)$, в другую оставшиеся слагаемые. Так как интервал изменения $r_{1}$ меньше $q$, то при фиксированном $r_{2}$ количество $r_{1}$, удовлетворяющих второму соотношению, не превосходит 1. Тем самым, оценивая в первом случае сумму тривиально, получим для нее неравенство $\ll R$. Пусть теперь

$$
W_{2}=\sum_{V<v \leqslant V_{2}} \chi\left(f_{1}(v)\right)
$$

при условии, что

$$
\begin{gathered}
r_{1} \neq r_{2}, \quad a\left(Q r_{1}+l_{1}\right)^{-1} \not \equiv b\left(Q r_{2}+l_{1}\right)^{-1}(\bmod q), \\
f_{1}(v)=\left(\left(Q r_{1}+l_{1}\right)\left(Q v+u_{1}\right)+a\right)\left(\left(Q r_{1}+l_{1}\right)\left(Q v+u_{1}\right)+b\right) \\
\times\left(\left(Q r_{2}+l_{1}\right)\left(Q v+u_{1}\right)+a\right)\left(\left(Q r_{2}+l_{1}\right)\left(Q v+u_{1}\right)+b\right), \quad V_{2} \leqslant 2 V .
\end{gathered}
$$

Обозначая через $A_{j}, B_{j}, j=1,2$, целые числа вида

$$
\begin{aligned}
& A_{j} \equiv\left(a\left(Q r_{j}+l_{1}\right)^{-1}+u_{1}\right) Q^{-1}(\bmod q), \\
& B_{j} \equiv\left(b\left(Q r_{j}+l_{1}\right)^{-1}+u_{1}\right) Q^{-1}(\bmod q),
\end{aligned}
$$

находим:

$$
\chi\left(f_{1}(v)\right)=\chi\left(\left(v+A_{1}\right)\left(v+A_{2}\right)\left(v+B_{1}\right)\left(v+B_{2}\right)\right) .
$$

Так как $A_{1} \not \equiv B_{1}, A_{2} \not \equiv B_{2}, A_{1} \not \equiv A_{2}, A_{1} \not \equiv B_{2}(\bmod q)$, то к $W_{2}$ применима оценка Хассе-Вейля, т. е.

$$
W_{2} \ll \sqrt{q}(\ln q)\left(V q^{-1}+1\right) .
$$

Тем самым, учитывая все оценки, получаем:

$$
\begin{aligned}
\left|W_{1}\right|^{2} & \ll \psi_{0}^{2} Q^{2} V\left(V R+\left(V q^{-1}+1\right) R^{2} \sqrt{q} \ln q\right) \\
& \ll \psi_{0}^{2} Q^{2} V^{2} R^{2}\left(R^{-1}+q^{-0.5} \ln q+V^{-1} \sqrt{q} \ln q\right) .
\end{aligned}
$$

По условиям леммы и рассматриваемого случая 2) имеем:

$$
R \asymp M Q^{-1}>q^{\omega}, \quad V \asymp U Q^{-1}>q^{0.5+0.05 \omega},
$$

т. e.

$$
\begin{aligned}
\left|W_{1}\right|^{2} & \ll \psi_{0}^{2} Q^{-2} M^{2} U^{2} q^{-0.05 \omega} \ln q \\
\left|W_{1}\right| & \ll \psi_{0} Q^{-1} M U q^{-0.05 \omega^{2}} .
\end{aligned}
$$


Cлучай 3). Так как $M U>Q^{2} q^{0.75+\omega}$ (по условию леммы) и, кроме того, $U<q^{0.5+0.05 \omega}, M<q^{0.5+0.05 \omega}$ (по условию рассматриваемого случая), то

$$
\begin{aligned}
& M>Q^{2} q^{0.75+\omega} U^{-1}>Q q^{0.25+0.95 \omega}, \\
& U>Q^{2} q^{0.75+\omega} M^{-1}>Q q^{0.25+0.95 \omega} .
\end{aligned}
$$

Суммирование по $u$ и $m$ представим в виде суммирования по арифметическим прогрессиям с разностью $Q$ :

$$
u=Q u_{1}+u_{2}, \quad m=Q m_{1}+m_{2},
$$

где

$$
\begin{gathered}
0<u_{2}<Q, \quad\left(u_{2}, Q\right)=1, \\
V=\left(U-u_{2}\right) Q^{-1}<u_{1} \leqslant\left(U_{1}-u_{2}\right) Q^{-1}=V_{1}, \quad V \gg q^{0.25+0.95 \omega} ;
\end{gathered}
$$

и

$$
\begin{gathered}
0<m_{2}<Q, \quad\left(m_{2}, Q\right)=1, \\
R=\left(M-m_{2}\right) Q^{-1}<m_{1} \leqslant\left(M_{1}-m_{2}\right) Q^{-1}=R_{1}, \\
R \gg q^{0.25+0.95 \omega} ; \quad R_{2}=\min \left(R_{1}, N u^{-1}\right) .
\end{gathered}
$$

Условие $m u \equiv l(\bmod Q)$ дает: $m_{2} u_{2} \equiv l(\bmod Q)$. Так как каждому $u_{2}$ отвечает одно $m_{2}$, то

$$
W_{1} \ll \psi_{0} Q \sum_{V<u_{1} \leqslant V_{1}}\left|\sum_{R<m_{1} \leqslant R_{2}} \chi\left(g\left(m_{1}, u_{1}\right)\right)\right|,
$$

где

$$
g\left(m_{1}, u_{1}\right)=\left(\left(Q m_{1}+m_{2}\right)\left(Q u_{1}+u_{2}\right)+a\right)\left(\left(Q m_{1}+m_{2}\right)\left(Q u_{1}+u_{2}\right)+b\right),
$$

$u_{2}$ и $m_{2}-$ фиксированные числа, $0<u_{2}<Q, 0<m_{2}<Q, m_{2} u_{2} \equiv l(\bmod Q)$, такие, что правая часть последнего неравенства принимает наибольшее значение. Опустим в последней двойной сумме слагаемые, для которых

$$
Q u_{1}+u_{2} \equiv 0(\bmod q)
$$

получим погрешность

$$
\ll \psi_{0} Q\left(V q^{-1}+1\right) R=\psi_{0} Q V R\left(q^{-1}+V^{-1}\right) .
$$

Для $g\left(m_{1}, u_{1}\right)$ находим соотношение:

$$
g\left(m_{1}, u_{1}\right) \equiv\left(m_{1}+A\right)\left(m_{1}+B\right) Q^{2}\left(Q u_{1}+u_{2}\right)^{2}(\bmod q),
$$

где

$$
\begin{gathered}
A=a^{\prime}\left(Q u_{1}+u_{2}\right)^{-1}+m_{2}^{\prime}, \quad B=b^{\prime}\left(Q u_{1}+u_{2}\right)^{-1}+m_{2}^{\prime}, \\
a Q^{-1} \equiv a^{\prime}(\bmod q), \quad b Q^{-1} \equiv b^{\prime}(\bmod q), \quad m_{2} Q^{-1} \equiv m_{2}^{\prime}(\bmod q) .
\end{gathered}
$$


Возьмем теперь $Y=\left[q^{0.1 \omega}\right], X=\left[R Y^{-1} q^{-0.05 \omega}\right] \gg q^{0.25+0.8 \omega}$ и с суммой $S$ по $m_{1}$ сделаем такие преобразования:

$$
\begin{aligned}
|S| & =\left|\sum_{R<m_{1} \leqslant R_{2}} \chi\left(g\left(m_{1}, u_{1}\right)\right)\right| \\
& =\left|\chi\left(Q^{2}\left(Q u_{1}+u_{2}\right)^{2}\right) \sum_{R<m_{1} \leqslant R_{2}} \chi\left(\left(m_{1}+A\right)\left(m_{1}+B\right)\right)\right| \\
& \leqslant(X Y)^{-1}\left|\sum_{R<m_{1} \leqslant R_{2}} \sum_{x=1}^{X} \sum_{y=1}^{Y} \chi\left(\left(m_{1}+x y+A\right)\left(m_{1}+x y+B\right)\right)\right|+O(X Y) .
\end{aligned}
$$

Следовательно, для $W_{1}$ получаем неравенство

$W_{1} \ll \psi_{0} Q(X Y)^{-1}$

$$
\begin{aligned}
& \quad \times \sum_{V<u_{1} \leqslant V_{1}} \sum_{R<m_{1} \leqslant 2 R} \sum_{x=1}^{X} \mid \sum_{y=1}^{Y} \chi\left(\left(\left(m_{1}+m_{2}^{\prime}\right) x^{-1}+a^{\prime}\left(Q u_{1}+u_{2}\right)^{-1} x^{-1}+y\right)\right. \\
& \left.\times\left(\left(m_{1}+m_{2}^{\prime}\right) x^{-1}+b^{\prime}\left(Q u_{1}+u_{2}\right)^{-1} x^{-1}+y\right)\right) \mid+\psi_{0} Q V R q^{-0.05 \omega} \\
& =\psi_{0} Q(X Y)^{-1} W_{2}+\psi_{0} Q V R q^{-0.05 \omega},
\end{aligned}
$$

где кратная сумма по $u_{1}, m_{1}, x, y$ обозначена символом $W_{2}$. Введем еще одно обозначение, именно, символом $J(\lambda, \mu)$ обозначим число решений следующей системы сравнений:

$$
\left\{\begin{array}{l}
\left(m_{1}+m_{2}^{\prime}\right) x^{-1} \equiv \lambda \\
\left(u_{1}+u_{2}^{\prime}\right) x^{-1} \equiv \mu
\end{array} \quad(\bmod q)\right.
$$

В этой системе $\lambda, \mu$ - произвольные, но фиксированные целые числа, а неизвестными системы являются числа $m_{1}, u_{1}, x$, причем $R<m_{1} \leqslant 2 R, V<$ $u_{1} \leqslant 2 V, 1 \leqslant x \leqslant X$. Тогда для $W_{2}$ получаем:

$$
W_{2}=\sum_{\lambda=0}^{q-1} \sum_{\mu=0}^{q-1} J(\lambda, \mu)\left|\sum_{y=1}^{Y} \chi\left(\left(\lambda+a^{\prime} \mu+y\right)\left(\lambda+b^{\prime} \mu+y\right)\right)\right| .
$$

Возьмем $k=\left[10 \omega^{-1}\right]+1$, возведем $W_{2}$ в степень $k$ и применим неравенство Гёльдера:

$$
W_{2}^{k} \leqslant\left(\sum_{\lambda=0}^{q-1} \sum_{\mu=0}^{q-1} J(\lambda, \mu)\right)^{k-1} \sum_{\lambda=0}^{q-1} \sum_{\mu=0}^{q-1} J(\lambda, \mu)\left|\sum_{y=1}^{Y} \chi\left(\left(\lambda+a^{\prime} \mu+y\right)\left(\lambda+b^{\prime} \mu+y\right)\right)\right|^{k} .
$$

$\mathrm{K}$ последней сумме по $\lambda$ и $\mu$ применим неравенство Коши:

$$
\begin{aligned}
W_{2}^{k} \leqslant & \left(\sum_{\lambda=0}^{q-1} \sum_{\mu=0}^{q-1} J(\lambda, \mu)\right)^{k-1} \sqrt{\sum_{\lambda=0}^{q-1} \sum_{\mu=0}^{q-1} J^{2}(\lambda, \mu)} \\
& \times \sqrt{\sum_{\lambda=0}^{q-1} \sum_{\mu=0}^{q-1}\left|\sum_{y=1}^{Y} \chi\left(\left(\lambda+a^{\prime} \mu+y\right)\left(\lambda+b^{\prime} \mu+y\right)\right)\right|^{2 k}} .
\end{aligned}
$$


Последняя кратная сумма (обозначим ее $W_{3}$ ) легко приводится к виду суммы $W$ леммы 3 главы I, в которой следует положить $\chi_{1}=\chi_{2}=\chi$. Действительно, пользуясь тем, что вместе с $\lambda$ произведение $\lambda \mu^{-1}$ пробегает полную систему вычетов ( $\mu$ и $q$ считаются взаимно простыми), $\mu^{-1}$ вместе с $\mu$ пробегает приведенную систему вычетов, получаем:

$$
\begin{aligned}
W_{3} & =\sum_{\lambda=0}^{q-1} \sum_{\mu=0}^{q-1}\left|\sum_{y=1}^{Y} \chi\left(\left(\lambda+a^{\prime} \mu+y\right)\left(\lambda+b^{\prime} \mu+y\right)\right)\right|^{2 k} \\
& =\sum_{\lambda=0}^{q-1} \sum_{\mu=1}^{q-1}\left|\sum_{y=1}^{Y} \chi\left(\left(\lambda+y \mu+a^{\prime}\right)\left(\lambda+y \mu+b^{\prime}\right)\right)\right|^{2 k}+O\left(q Y^{2 k}\right) \\
& =\sum_{\lambda=1}^{q} \sum_{\mu=1}^{q}\left|\sum_{y=1}^{Y} \chi\left(\left(\lambda+y \mu+a^{\prime}\right)\left(\lambda+y \mu+b^{\prime}\right)\right)\right|^{2 k}+O\left(q Y^{2 k}\right) .
\end{aligned}
$$

Следовательно,

$$
W_{3} \ll Y^{k} q^{2}+Y^{2 k} q .
$$

Далее, сумма значений $J(\lambda, \mu)$ по всем $\lambda$ и $\mu$ равняется количеству всех возможных наборов чисел $m_{1}, u_{1}, x$, т. е. « $R V X$. Осталось оценить сумму значений $J^{2}(\lambda, \mu)$, которую обозначим буквой $\sigma$. Очевидно, что $\sigma$ равняется числу решений следующей системы сравнений:

$$
\begin{aligned}
& \left\{\begin{array}{l}
\left(m_{1}+m_{2}^{\prime}\right) x^{-1} \equiv\left(\bar{m}_{1}+m_{2}^{\prime}\right) \bar{x}^{-1} \\
\left(u_{1}+u_{2}^{\prime}\right) x^{-1} \equiv\left(\bar{u}_{1}+u_{2}^{\prime}\right) \bar{x}^{-1}
\end{array} \quad(\bmod q),\right. \\
& R<m_{1}, \bar{m}_{1} \leqslant 2 R, \quad V<u_{1}, \bar{u}_{1} \leqslant 2 V, \quad 1 \leqslant x, \bar{x} \leqslant X .
\end{aligned}
$$

Преобразуем нашу систему, освобождаясь от отрицательных степеней $x$ и группируя слагаемые:

$$
\left\{\begin{array}{l}
m_{1} \bar{x}-\bar{m}_{1} x \equiv m_{2}^{\prime}(x-\bar{x}) \\
u_{1} \bar{x}-\bar{u}_{1} x \equiv u_{2}^{\prime}(x-\bar{x})
\end{array} \quad(\bmod q) .\right.
$$

Заменяя $x$ на $\bar{x}+h,|h| \leqslant X$, получим:

$$
\left\{\begin{array}{l}
\left(m_{1}-\bar{m}_{1}\right) \bar{x} \equiv\left(\bar{m}_{1}+m_{2}^{\prime}\right) h \\
\left(u_{1}-\bar{u}_{1}\right) \bar{x} \equiv\left(\bar{u}_{1}+u_{2}^{\prime}\right) h
\end{array} \quad(\bmod q) .\right.
$$

Заметим, что левые (а значит, и правые) части этой системы сравнений кратны $q$ только при $m_{1}=\bar{m}_{1}, u_{1}=\bar{u}_{1}$. Но тогда получим $h=0$, так как $\left(m_{1}+m_{2}^{\prime}\right) Q \equiv Q m_{1}+m_{2}(\bmod Q)$ и $\left(Q m_{1}+m_{2}, q\right)=1$ по условию леммы. Таким образом, в этом случае число решений системы $R V X$. Оценим теперь число решений системы при условии, что хотя бы одна из левых частей не кратна $q$. Фиксируем $\bar{m}_{1}, \bar{u}_{1}, h$. Количество таких наборов $\ll R V X$. Так как $m_{1}, \bar{m}_{1} \leqslant R \leqslant q^{0.5+0.05 \omega}, u, \bar{u}_{1} \leqslant q^{0.5+0.05 \omega}, \bar{x} \leqslant X \ll R Y^{-1} q^{-0.05 \omega} \ll q^{0.5-0.1 \omega}$, то

$$
\left|\left(m_{1}-\bar{m}_{1}\right) \bar{x}\right| \ll q^{1-0.95 \omega}, \quad\left|\left(u_{1}-\bar{u}_{1}\right) \bar{x}\right| \ll q^{1-0.95 \omega},
$$


и система сравнений эквивалентна следующей системе уравнений:

$$
\left\{\begin{array}{l}
\left(m_{1}-\bar{m}_{1}\right) \bar{x}=\alpha \\
\left(u_{1}-\bar{u}_{1}\right) \bar{x}=\beta
\end{array}\right.
$$

где $\alpha$ и $\beta$ - целые числа, $|\alpha| \leqslant q,|\beta| \leqslant q$, причем либо $\alpha$, либо $\beta$ отлично от нуля. Число решений этой системы уравнений $\ll q^{\varepsilon}$. Тем самым для $\sigma$ находим оценку:

$$
\sigma \ll R V X q^{\varepsilon} .
$$

Подставим найденные оценки в неравенство для $W_{2}^{k}$ :

$$
\begin{aligned}
W_{2}^{k} & \ll(R V X)^{k-1} \sqrt{R V X q^{\varepsilon}} \sqrt{Y^{k} q^{2}+Y^{2 k} q} \\
& \ll(R V X)^{k-0.5} Y^{k} q^{1+\varepsilon}\left(Y^{-k}+q^{-1}\right)^{0.5} .
\end{aligned}
$$

Теперь, вспоминая, что

$$
\begin{gathered}
R \asymp M Q^{-1}, \quad V \asymp U Q^{-1}, \quad Y \asymp q^{0,1 \omega}, \\
X \asymp R Y^{-1} q^{-0.05 \omega}, \quad M U>Q^{2} q^{0.75+\omega}, \quad k=\left[10 \omega^{-1}\right]+1>10 \omega^{-1},
\end{gathered}
$$

легко находим:

$$
\begin{gathered}
Y^{k} \asymp q^{0.1 \omega k}>q, \quad R V X \asymp M U Q^{-2} M Q^{-1} q^{-0.15 \omega} \gg q^{1+1.8 \omega}, \\
W_{2}^{k} \ll(X Y R V)^{k}(R V X)^{-0.5} q^{0.5+\omega}, \quad W_{2}^{k} \ll(X Y R V)^{k} q^{-0.8 \omega}, \\
W_{2} \ll X Y R V q^{-0.05 \omega^{2}}, \quad W_{1} \ll \psi_{0} Q^{-1} M U q^{-0.05 \omega^{2}} .
\end{gathered}
$$

Отсюда следует утверждение леммы.

ЛЕмма 2. Пусть $\psi_{1}(u)$ и $\psi_{2}(m)$ - произвольные комплекснозначные функции, причем $\left|\psi_{1}(u)\right| \leqslant \psi_{1},\left|\psi_{2}(m)\right| \leqslant \psi_{2}, U>Q q^{0.5+\omega}, M>Q q^{\omega}, M<$ $M_{1} \leqslant 2 M, U<U_{1} \leqslant 2 U$,

$$
W_{4}=\sum_{\substack{M<m \leqslant M_{1} \\ m u \leqslant N, m u \equiv l(\bmod Q)}} \sum_{\substack{U<U_{1} \\ m, m}} \psi_{2}(m) \chi(f(u m)) .
$$

Тогда для $\left|W_{4}\right|$ справедлива следующая оценка:

$$
\left|W_{4}\right| \leqslant c_{2}(\omega) \psi_{1} \psi_{2} Q^{-1} M U q^{-0.4 \omega} .
$$

ДокАЗАТЕльство. Эта лемма, по существу, является несложным обобщением рассмотренного в лемме 1 случая 2). Представим $m$ и $u$ в виде

$$
m=Q m_{1}+m_{2}, \quad u=Q u_{1}+u_{2},
$$

где

$$
0<m_{2}<Q, \quad R=\left(M-m_{2}\right) Q^{-1}<m_{1} \leqslant\left(M_{1}-m_{2}\right) Q^{-1}=R_{1}
$$

И

$$
0<u_{2}<Q, \quad V=\left(U-u_{2}\right) Q^{-1}<u_{1} \leqslant\left(U_{1}-u_{2}\right) Q^{-1}=V_{1}
$$


Так как $m u \equiv l(\bmod Q)$, то $m_{2} u_{2} \equiv l(\bmod Q)$. Переходя к неравенствам, будем иметь:

$$
\begin{aligned}
\left|W_{4}\right| \leqslant & \sum_{\substack{0<m_{2}<Q \\
m_{2} u_{2} \equiv l(\bmod Q)}} \mid \sum_{\substack{0<u_{2}<Q \\
\left(Q m_{1}+m_{2}\right)\left(Q u_{1}+u_{2}\right) \leqslant N}} \sum_{\substack{V<u_{1} \leqslant V_{1} \\
\left(Q m_{1}\right.}} \psi_{1}\left(Q m_{1}+m_{2}\right) \psi_{2}\left(Q u_{1}+u_{2}\right) \\
& \times \chi\left(f\left(\left(Q m_{1}+m_{2}\right)\left(Q u_{1}+u_{2}\right)\right)\right) \mid \\
\leqslant & \psi_{2} Q \sum_{V<u_{1} \leqslant V_{1}}\left|\sum_{R<m_{1} \leqslant R_{2}} \psi_{1}\left(Q m_{1}+m_{2}\right) \chi\left(f\left(\left(Q m_{1}+m_{2}\right)\left(Q u_{1}+u_{2}\right)\right)\right)\right|,
\end{aligned}
$$

где $R_{2}=\min \left(R_{1}, N\left(Q u_{1}+u_{2}\right)^{-1}\right), m_{2}, u_{2}$ - уже фиксированные натуральные числа. Возведем обе части неравенства в квадрат и применим неравенство Коши:

$$
\left|W_{4}\right|^{2} \ll \psi_{2}^{2} Q^{2} V \sum_{V<u_{1} \leqslant 2 V}\left|\sum_{R<m_{1} \leqslant R_{2}}\right|^{2}=\psi_{2}^{2} Q^{2} V W_{5},
$$

где

$$
\begin{aligned}
W_{5} & =\sum_{R<m_{1}, m_{1}^{\prime} \leqslant R_{1}} \psi_{1}\left(Q m_{1}+m_{2}\right) \overline{\psi_{1}\left(Q m_{1}^{\prime}+m_{2}\right)} W_{6}\left(m_{1}, m_{1}^{\prime}\right), \\
W_{6}\left(m_{1}, m_{1}^{\prime}\right) & =\sum_{V<u_{1} \leqslant V_{2}} \chi\left(f\left(\left(Q m_{1}+m_{2}\right)\left(Q u_{1}+u_{2}\right)\right) f\left(\left(Q m_{1}^{\prime}+m_{2}\right)\left(Q u_{1}+u_{2}\right)\right)\right) .
\end{aligned}
$$

Следовательно,

$$
\left|W_{5}\right| \leqslant \psi_{1}^{2} \sum_{R<m_{1}, m_{1}^{\prime} \leqslant 2 R}\left|W_{6}\left(m_{1}, m_{1}^{\prime}\right)\right| .
$$

Но последнюю кратную сумму мы уже оценили в лемме 1, рассматривая случай 2). Таким образом, будем иметь:

$$
\begin{aligned}
\left|W_{5}\right| & \ll \psi_{1}^{2}\left(\left(1+R q^{-1}\right) R V+R^{2}\left(V q^{-1}+1\right) \sqrt{q} \ln q\right) \\
& \ll \psi_{1}^{2} R^{2} V^{2}\left(R^{-1} V^{-1}+q^{-0.5} V^{-1}+q^{0.5} V^{-2}\right) \ln q \\
\left|W_{4}\right|^{2} & \ll \psi_{1}^{2} \psi_{2}^{2} Q^{2} R^{2} V^{2}\left(R^{-1}+q^{-0.5}+q^{0.5} V^{-1}\right) \ln q .
\end{aligned}
$$

По условию леммы $R \gg q^{\omega}, V \gg q^{0.5+\omega}$, поэтому

$$
\begin{aligned}
\left|W_{4}\right|^{2} & \ll \psi_{1}^{2} \psi_{2}^{2} Q^{2} R^{2} V^{2} q^{-\omega} \ln q ; \\
\left|W_{4}\right| & \ll \psi_{1} \psi_{2} Q R V q^{-0.4 \omega} \ll \psi_{1} \psi_{2} Q^{-1} M U q^{-0.4 \omega},
\end{aligned}
$$

что и требовалось доказать.

\section{Глава IV. Суммы характеров с весами}

\section{$\S 1$. Линейные суммы с весами}

При целом числе $l$ рассмотрим следующую сумму $T(X)$,

$$
T(X)=\sum_{n \leqslant X} \tau_{k}(n) \chi(n+l) .
$$

Нахождение нетривиальной оценки для $|T(X)|$ при $X=X(q)$, любых значе- 
ниях $k$ и $l \equiv 0(\bmod q)$ в настоящее время проблематично. Однако аналогичная задача при $l \not \equiv 0(\bmod q)$ может быть решена (см. [44]).

ТЕОРема 1. Пусть $\omega$ - произвольное положительное число, не превосходящее $0.25,(l, q)=1, q^{0.5+\omega} \leqslant X \leqslant q^{10}$. Тогда для $|T(X)|$ справедлива следующая оценка:

$$
|T(X)| \ll X q^{-0.011 \omega^{2}} \exp \left(\frac{8 k \ln X}{\ln \ln X}\right),
$$

где постоянная в знаке «зависит только от $\omega$.

Из теоремы легко получается

СлЕДСтвиЕ 1. Пусть а - произвольное натуральное число, $q \geqslant q_{1}(a)$, $X$ удовлетворяет условиям теоремы. Пусть, далее, суммы $T_{1}(X), T_{2}(X)$, $T_{3}(X)$, определяются равенствами

$$
\begin{gathered}
T_{1}(X)=\sum_{n \leqslant X}^{\prime} \tau_{k}(n+a), \quad T_{2}(X)=\sum_{n \leqslant X}^{\prime \prime} \tau_{k}(n+a), \\
T_{3}(X)=\sum_{n \leqslant X}^{\prime \prime \prime} \tau_{k}(n+a),
\end{gathered}
$$

где штрих в сумме $T_{1}(X)$ и два штриха в сумме $T_{2}(X)$ означают суммирование по числам $n$, которые являются, соответственно, квадратичными вычетами и невычетами по модулю $q$, а три штриха в сумме $T_{3}(X)$ означает суммирование по числам $n$, которые являются первообразными корнями по модулю q. Тогда справедливы следующие асимптотические формулы:

$$
\begin{aligned}
& T_{1}(X)=\frac{1}{2} A_{k}(X)+O\left(X q^{-0.01 \omega^{2}} \exp \left(\frac{8 k \ln X}{\ln \ln X}\right)\right), \\
& T_{2}(X)=\frac{1}{2} A_{k}(X)+O\left(X q^{-0.01 \omega^{2}} \exp \left(\frac{8 k \ln X}{\ln \ln X}\right)\right), \\
& T_{3}(X)=\frac{\varphi(q-1)}{q-1} A_{k}(X)+O\left(X q^{-0.01 \omega^{2}} \exp \left(\frac{8 k \ln X}{\ln \ln X}\right)\right),
\end{aligned}
$$

2วe

$$
A_{k}(X)=\sum_{n \leqslant X} \tau_{k}(n)=X P_{k}(\ln X)+O\left(X^{\frac{k-1}{k+1}+\varepsilon}\right)
$$

u $P_{k}(t)$ - многочлен степени $k-1$ от $t$.

ДоКАЗАТЕЛЬСТво ТЕОРЕМЫ 1. Имеем равенство:

$$
T(X)=\sum_{x_{1} \cdots x_{k} \leqslant X} \chi\left(x_{1} \cdots x_{k}+l\right) .
$$

Каждая из переменных суммирования $x_{1}, \ldots, x_{k}$ пробегает значения целых чисел интервала $[1, X]$. Эти значения связаны соотношением $x_{1} \cdots x_{k} \leqslant X$. Разобъем каждый из таких интервалов на $\ll \ln X$ интервалов вида $(Y, 2 Y]$. Соответственно этому разбиению сумма $T(X)$ разобьется на $\ll(2 \ln X)^{k}$ сумм $W$ вида

$$
W=\sum_{X_{1}<x_{1} \leqslant 2 X_{1}} \cdots \sum_{\substack{X_{k}<x_{k} \leqslant 2 X_{k} \\ x_{1} \cdots x_{k} \leqslant X}} \chi\left(x_{1} \cdots x_{k}+l\right) .
$$


Рассмотрим одну из таких сумм. Достаточно рассмотреть случай

$$
X_{1} \cdots X_{k} \geqslant X q^{-0.25 \omega}
$$

Никак не нарушая общности, будем считать, что $1 \leqslant X_{1} \leqslant \cdots \leqslant X_{k}$.

Если $X_{k} \geqslant q^{0.25+0.25 \omega}$, то по лемме 3 главы II имеем оценку

$$
|W| \ll q^{\varepsilon} X q^{-0.031 \omega^{2}} \max _{n \leqslant X} \tau_{k}(n) .
$$

Если $q^{0.25 \omega} \leqslant X<q^{0.25+0.25 \omega}$, то, вводя обозначения $x_{k}=u, x_{1} \cdots x_{k-1}=v$, $\psi(v)$ - число решений в числах $x_{1}, \ldots, x_{k-1}$ уравнения $x_{1} \cdots x_{k-1}=v, U=X_{k}$, $V=X_{1} \cdots X_{k-1}$, получим:

$$
W=\sum_{V<v \leqslant 2^{k} V} \sum_{\substack{U<u \leqslant 2 U \\ u v \leqslant X}} \psi(v) \chi(u v+l) .
$$

Легко проверить следующие соотношения:

$$
\begin{gathered}
q^{0.25 \omega} \leqslant U<q^{0.25+0.25 \omega} \leqslant q^{0.5-0.125}, \quad U V>X q^{-0.25 \omega} \geqslant q^{0.5+0.75 \omega}, \\
V U^{-1} \geqslant q^{0.5+0.75 \omega} U^{-2} \geqslant q^{0.25 \omega} .
\end{gathered}
$$

Следовательно, к $W$ можно применить лемму 7 главы II, полагая в ней $Q=1$. После простых вычислений получаем:

$$
|W| \ll q^{\varepsilon} \cdot 2^{k} X q^{-0.015 \omega^{2}} \max _{n \leqslant X} \tau_{k-1}(n) .
$$

Наконец, если $X_{k}<q^{0.25 \omega}$, то, так как

$$
X_{1} \cdots X_{k} \geqslant X q^{-0.25 \omega}
$$

найдутся номера $j_{1}, \ldots, j_{s-1}, j_{s}$ такие, что

$$
X_{j_{1}} \cdots X_{j_{s-1}}<q^{0.5+0.25 \omega}<X_{j_{1}} \cdots X_{j_{s-1}} X_{j_{s}}<q^{0.5+0.5 \omega} .
$$

Обозначая символами $\psi_{1}(u)$ и $\psi_{2}(v)$ соответственно числа решений уравнений $x_{j_{1}} \cdots x_{j_{s}}=u, x_{1} \cdots x_{k} u^{-1}=v$, в числах $x_{1}, \ldots, x_{k}$, видим, что $\psi_{1}(u) \leqslant \tau_{s}(u)$, $\psi_{2}(v) \leqslant \tau_{k-s}(v)$. Кроме того, введенные величины $u$ и $v$ удовлетворяют неравенствам

$$
q^{0.5+0.25 \omega}<u<q^{0.5+0.5 \omega}, \quad v \geqslant q^{0.25 \omega} .
$$

Разбивая интервалы суммирования по $u$ и $v$ на интервалы вида $(U, 2 U],(V, 2 V]$, получим « $(\ln X)^{2}$ сумм, к каждой из которых можно применить лемму 5 главы II, полагая в ней $Q=1$; получим:

$$
|W| \ll 2^{k} X q^{-0.015 \omega^{2}}\left(\max _{n \leqslant X} \tau_{k}(n)\right)^{2} .
$$

Из оценок (23)-(25) следует утверждение теоремы 1. 


\section{§ 2. Нелинейные суммы символов Лежандра с весами}

В этом параграфе считаем $k$ фиксированным натуральным числом, $k \geqslant 2$; $q \geqslant q_{1}(k) ; \omega \in\left(0, \frac{1}{4}\right) ; a$ и $b-$ целые числа с условием $a b(a-b) \not \equiv 0(\bmod q)$; постоянные в знаках « и $O$ зависят только от $\omega$.

Подобно тому, как доказывалась теорема 1, но только с привлечением лемм главы III, доказывается следующая теорема.

ТЕОРЕма 2. При любом $X$ из промежутка

$$
q^{0.75+\omega} \leqslant X \leqslant q^{2}
$$

справедлива оченка:

$$
\left|\sum_{n \leqslant X} \tau_{k}(n)\left(\frac{(n+a)(n+b)}{q}\right)\right| \ll X^{1-0.01 \omega^{2}} .
$$

СлЕДСТВиЕ 2. Пусть $s_{1}= \pm 1, s_{2}= \pm 1$,

$$
S=\sum_{n \leqslant X}^{\prime} \tau_{k}(n)
$$

где штрих означает суммирование по таким $n \leqslant X$, для которых

$$
\left(\frac{n+a}{q}\right)=s_{1}, \quad\left(\frac{n+b}{q}\right)=s_{2} .
$$

Тогда при любом $X$ из промежутка $q^{0.75+\omega} \leqslant X \leqslant q^{2}$ справедлива асимптотическая формула:

$$
S=\frac{1}{4} A_{k}(X)+O\left(X q^{-0.01 \omega^{2}} \exp \left(8 k \frac{\ln X}{\ln \ln X}\right)\right),
$$

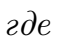

$$
A_{k}(X)=\sum_{n \leqslant X} \tau_{k}(n)=X P_{k-1}(\ln X)+O\left(X^{\frac{k-1}{k+1}} \ln ^{k} X\right) .
$$

Доказательство теоремы и следствия см. в [45].

\section{§ 3. Нелинейные суммы характеров с весами}

В этом параграфе оцениваются суммы

$$
\begin{aligned}
S_{1} & =\sum_{n \leqslant X} \tau_{k}(n) \chi(n+a), & S_{2} & =\sum_{n \leqslant X} \tau_{k}(n) \chi((n+a)(n+b)), \\
R_{1} & =\sum_{x^{2}+y^{2} \leqslant X} \chi\left(x^{2}+y^{2}+a\right), & R_{2} & =\sum_{x^{2}+y^{2} \leqslant X} \chi_{1}\left(x^{2}+y^{2}+a\right) \chi_{2}\left(x^{2}+y^{2}+b\right) .
\end{aligned}
$$

Здесь, как и раньше, $\chi, \chi_{1}, \chi_{2}$ - неглавные характеры Дирихле по простому модулю $q, a b(a-b) \not \equiv 0(\bmod q)$. Основной проблемой при изучении указанных сумм является проблема нетривиальной оценки их модуля при возможно меньших значениях $X$ по отношению к $q$. Здесь получены оценки со степенным понижением при $X$ значительно меньших, чем соответствующие $X$ в 11 и $\S 2$ (см. [46]). 
Tеорема 3. Пусть $X \geqslant q^{\frac{1}{2}-\frac{1}{2(k+1)}+\delta}, 0<\delta<\frac{2}{3(k+1)}, 0<|a| \leqslant \sqrt{q}$. Тогда для $\left|S_{1}\right|$ справедлива оценка:

$$
\left|S_{1}\right| \ll X q^{-0.02 \delta^{2}}
$$

Теорема 4. Пусть $X \geqslant q^{\frac{1}{3}+\delta}, 0<|a| \leqslant \sqrt{q}$. Тогда для $\left|R_{1}\right|$ справедлива следующая оценка:

$$
\left|R_{1}\right| \ll X q^{-0.02 \delta^{2}}
$$

СлЕДСТвИЕ 3. При обозначениях следствия 1 и при выполнении условий теоремы 3 справедливы следуюшие асимптотические формулы:

$$
\begin{aligned}
& T_{1}(X)=\frac{1}{2} A_{k}(X)+O\left(X q^{-0.02 \delta^{2}}\right) \\
& T_{2}(X)=\frac{1}{2} A_{k}(X)+O\left(X q^{-0.02 \delta^{2}}\right) \\
& T_{3}(X)=\frac{\varphi(q-1)}{q-1} A_{k}(X)+O\left(X q^{-0.01 \delta^{2}}\right) .
\end{aligned}
$$

СледствиЕ 4. Пусть $K(X)$ - количество иельх точек в круге $x^{2}+y^{2} \leqslant X$ таких, что числа $x^{2}+y^{2}+$ а являются квадратичными вычетами (невычетами) по модулю q. Тогда при выполнении условий теоремы 4 справедлива следующая асимптотическая формула:

$$
K(X)=\frac{\pi}{2} X+O\left(X q^{-0.02 \delta^{2}}\right) .
$$

СлеДСтвиЕ 5. Пусть $G(X)$ - количество иелых точек в круге $x^{2}+y^{2} \leqslant X$ таких, что числа $x^{2}+y^{2}+$ а являются первообразными корнями по модулю $q$. Тогда при выполнении условий теоремы 4 справедлива следующая асимптотическая формула:

$$
G(X)=\frac{\varphi(q-1)}{q-1} X+O\left(X q^{-0.01 \delta^{2}}\right) .
$$

Теорема 5. Пусть $X \geqslant q^{\frac{2}{3}+\delta}$. Тогда для $\left|S_{2}\right|$ справедлива следующая оценка:

$$
\left|S_{2}\right| \ll X q^{-0.02 \delta^{2}}
$$

Теорема 6. Пусть $X \geqslant q^{\frac{2}{3}+\delta}$. Тогда для $\left|R_{2}\right|$ справедлива следующая оценка:

$$
\left|R_{2}\right| \ll X q^{-0.02 \delta^{2}}
$$

СлЕДСТвиЕ 6. Пусть $0<|a| \leqslant \sqrt{q}, 0<|b| \leqslant \sqrt{q}, s_{1}= \pm 1, s_{2}= \pm 1$,

$$
V(X)=\sum_{n \leqslant X}^{\prime} \tau(n),
$$

где штрих в сумме означает суммирование по таким натуральным числам $n$, для которых выполняются равенства

$$
\left(\frac{n+a}{q}\right)=s_{1}, \quad\left(\frac{n+b}{q}\right)=s_{2} .
$$


Тогда при выполнении условий теоремы 5 справедлива следующая асимптотическая формула:

$$
V(X)=\frac{1}{4} X(\ln X+2 \gamma-1)+O\left(X q^{-0.01 \delta^{2}}\right),
$$

где $\gamma$ - постоянная Эйлера.

СлЕДСТвиЕ 7. Пусть $0<a \leqslant \sqrt{q}, 0<b \leqslant \sqrt{q}$,

$$
G(X)=\sum_{n \leqslant X}^{\prime} \tau(n)
$$

где штрих в сумме означает суммирование по таким натуральным числам $n \leqslant X$, для которых числа $n+a$ u $n+b$ одновременно являются первообразными корнями по модулю q. Тогда при выполнении условий теоремы 5 справедлива следующая асимптотическая формула:

$$
G(X)=\left(\frac{\varphi(q-1)}{q-1}\right)^{2} X(\ln X+2 \gamma-1)+O\left(X q^{-0.01 \delta^{2}}\right)
$$

где $\gamma$ - постоянная Эйлера.

СЛЕДСТвиЕ 8. Пусть $0<a, b \leqslant \sqrt{q}, s_{1}= \pm 1, s_{2}= \pm 1, K(X)$ - количество целых точек $(x, y)$ в круге $x^{2}+y^{2} \leqslant X$ таких, что

$$
\left(\frac{x^{2}+y^{2}+a}{q}\right)=s_{1}, \quad\left(\frac{x^{2}+y^{2}+b}{q}\right)=s_{2} .
$$

Тогда при выполнении условий теоремы 6 справедлива следующая асимптотическая формула:

$$
K(X)=\frac{\pi}{4} X+O\left(X q^{-0.01 \delta^{2}}\right) .
$$

СлЕДСТвИЕ 9. Пусть $0<a, b \leqslant \sqrt{q}, G(X)$ - количество целых точек $(x, y)$ в круге $x^{2}+y^{2} \leqslant X$ таких, что числа $x^{2}+y^{2}+a u x^{2}+y^{2}+b$ являются одновременно первообразными корнями по модулю q. Тогда при выполнении условий теоремы 6 справедлива следующая асимптотическая формула:

$$
G(X)=\pi\left(\frac{\varphi(q-1)}{q-1}\right)^{2} X+O\left(X q^{-0.01 \delta^{2}}\right) .
$$

\section{Глава V. Распределение значений характеров Дирихле на аддитивных последовательностях}

\section{§ 1. Распределение степенных вычетов и невычетов в аддитивных последовательностях}

Аддитивной последовательностью называется последовательность натуральных чисел $C$, которая является суммою двух других последовательностей натуральных чисел $A$ и $B$, т. е. для любого $c \in C$ существуют $a \in A$ и $b \in B$ такие, что $c=a+b$, и, наоборот, $a+b \in C$ при любых $a \in A, b \in B$. 
Здесь мы будем заниматься проблемами распределения степенных вычетов и невычетов в аддитивных последовательностях $C=A+B$, где $A=P$ - множество простых чисел, $B=U$ - произвольное множество натуральных чисел. Основной характеристикой $U$ будет служить функция $g(x)=g(x ; U)$ - количество чисел $u \in U$ таких, что $u \leqslant x$.

В главе II рассматривалась аналогичная проблема в случае, когда $U$, грубо говоря, состояло из одного числа. Рассмотрение густых множеств $U$ дает возможность получить более тонкие результаты, именно, удается нетривиально оценить значительно более короткие суммы характеров, что позволяет, в свою очередь, получить соответствующие результаты в теории степенных вычетов и невычетов, первообразных корней и др. (см. [40]).

Tеорема 1. Рассмотрим сумму

$$
S=S(N, M)=\sum_{p \leqslant N} \sum_{u \leqslant M} \chi(p+u),
$$

где $p \in P, u \in U$, а числа $N$ и $M$ таковы, что

$$
1 \leqslant M N<q .
$$

Тогда при любом натуральном числе $k \geqslant \frac{\ln q}{\ln N}$ для $|S|$ справедлива оченка:

$$
|S| \ll \pi(N) g(M) \Delta, \quad \Delta=\left(\frac{q^{\frac{1}{2}+\frac{1}{2 k}}}{N g(M)}\right)^{\gamma / k},
$$

где $\gamma>0$ - абсолютная константа, а постоянная в знаке « зависит только om $k$.

ТЕОРема 2. При любом натуральном числе $k \geqslant \min \left(\frac{\ln q}{\ln N}, \frac{\ln q}{\ln M}\right)$ справедлива оченка

$$
\begin{gathered}
\left|\sum_{p \leqslant N} \sum_{p^{\prime} \leqslant M} \chi\left(p+p^{\prime}\right)\right| \ll \pi(N) \pi(M) \Delta, \\
\Delta=\left(q^{\frac{1}{2}+\frac{1}{2 k}}(N M)^{-1}\right)^{\gamma / k},
\end{gathered}
$$

где $p, p^{\prime} \in P, \gamma>0$ - абсолютная константа, а постоянная в знаке «зависит только от $k$.

Теорема 3. Пусть $N \geqslant q^{0.25+\varepsilon_{1}}, M \geqslant q^{0.25+\varepsilon_{1}} ;$ тогда справедлива оценка

$$
\left|\sum_{p \leqslant N} \sum_{p^{\prime} \leqslant M} \chi\left(p+p^{\prime}\right)\right| \ll \pi(N) \pi(M) q^{-\gamma_{1} \varepsilon_{1}^{2}},
$$

где $\gamma_{1}>0$ - абсолютная константа, а постоянная в знаке « зависит только om $\varepsilon_{1}$.

СЛЕДСТВИЕ 1. Существует $q_{1}=q_{1}\left(\varepsilon_{1}\right)$ maкое, что при $q \geqslant q_{1}$ на отрезке $\left[1, q^{0.25+\varepsilon_{1}}\right]$ содержится $c_{2} q^{0.25+\varepsilon_{1}}$ и $c_{3} q^{0.25+\varepsilon_{1}}$ квадратичных вычетов и соответственно квадратичных невычетов по модулю $q$ вида $p+p^{\prime}$, где $p$ и $p^{\prime}-$ простые числа, $c_{2}>0, c_{3}>0-$ константы.

Аналогично формулируются утверждения о вычетах и невычетах произвольной степени, а также о первообразных корнях по модулю $q$, имеющих вид $p+p^{\prime}$. 


\section{§ 2. Распределение значений характеров Дирихле на аддитивных последовательностях}

Рассмотрим двойную сумму $W$,

$$
W=\sum_{x \in A} \sum_{y \in B} \chi(x+y),
$$

где $\chi$ - неглавный характер по модулю $q, A$ и $B$ - произвольные множества натуральных чисел, не превосходящих $q$, причем

$$
\|A\| \geqslant q^{\delta}, \quad\|B\| \geqslant q^{0.5+\delta}
$$

где $\delta$ - произвольное положительное число, не превосходящее 0.5 , а $\|A\|$ и $\|B\|$ количество чисел множеств $A$ и $B$ соответственно. Подобно тому, как доказывалась теорема $1 \S 1$, получаем:

$$
|W| \ll\|A\|\|B\| q^{-0.05 \delta^{2}}
$$

где постоянная в знаке «зависит только от $\delta$.

В приложениях часто про множества $A$ и $B$ известно, что они сосредоточены на некоторых промежутках вида $(a, a+X]$ и $(b, b+Y]$, длины которых близки к количеству элементов множеств $A$ и $B$, другими словами,

$$
X \approx\|A\|, \quad Y \approx\|B\|
$$

Кроме того, слагаемые $x$ и $y$ в (26) могут повторяться. Так появляются суммы $W_{1}$,

$$
W_{1}=\sum_{x \in X} \sum_{y \in Y} \psi_{1}(x) \psi_{2}(y) \chi(x+y+a),
$$

где $0 \leqslant a<q, \psi_{1}(x), \psi_{2}(y)$ - произвольные комплекснозначные функции с условием $\left|\psi_{1}(x)\right| \leqslant \psi_{1},\left|\psi_{2}(y)\right| \leqslant \psi_{2}$. Тривиальная оценка $\left|W_{1}\right|$ такова:

$$
\left|W_{1}\right| \leqslant X Y \psi_{1} \psi_{2}
$$

Проблема состоит в том, чтобы получить оценку вида

$$
\left|W_{1}\right| \leqslant X Y \psi_{1} \psi_{2} \Delta
$$

где $\Delta=\Delta(q ; X, Y) \rightarrow 0$ при $q \rightarrow+\infty$.

Аналогом оценки (27) является оценка, которая содержится в следующей теореме.

ТЕОрема 4. Пусть $X \geqslant q^{\delta}, Y \geqslant q^{0.5+\delta}, q \geqslant q_{1}(\delta)$. Тогда для $\left|W_{1}\right|$ справедлива оченка

$$
\left|W_{1}\right| \leqslant X Y \psi_{1} \psi_{2} q^{-0.4 \delta} \leqslant X Y \psi_{1} \psi_{2} q^{-0.05 \delta^{2}}
$$

В нижеследующей теореме 5 получен результат при более слабых ограничениях на $X$ и $Y$, чем те, которые содержатся в теореме 4 (см. [41]). 
Tеорема 5. Пусть $0<\delta<0.5, q \geqslant q_{1}(\delta), X \geqslant q^{\delta}, \sqrt{X} Y \geqslant q^{0.5+\delta}$. Тогда для $\left|W_{1}\right|$ справедлива следующая ощенка:

$$
\left|W_{1}\right| \leqslant X Y \psi_{1} \psi_{2} q^{-0.05 \delta^{2}} .
$$

СлЕДСтвиЕ 2. Если $X \asymp Y, Y \geqslant q^{\frac{1}{3}+\delta}, m o$

$$
\left|W_{1}\right| \leqslant X Y \psi_{1} \psi_{2} q^{-0.05 \delta^{2}} \text {. }
$$

Основная идея доказательства теоремы совпадает с идеей доказательства леммы 1 главы III, в которой можно считать $Q=1$.

\section{Глава VI. Некоторые специальные вопросы теории характеров, нерешенные проблемы и гипотезы}

В 1926 г. И. М. Виноградов [55] (см. также [56]), пользуясь своей оценкой (13) и соображениями типа решета Бруна, доказал, что при любом $\varepsilon>0$ и $q \geqslant q_{1}(\varepsilon)$ наименьший квадратичный невычет по модулю $q$ не превосходит $N_{\min }$, где

$$
N_{\min } \leqslant q^{\frac{1}{2 \sqrt{e}}+\varepsilon}
$$

Тогда же возникла знаменитая гипотеза Виноградова, что

$$
N_{\min } \leqslant q^{\varepsilon}
$$

В 1942 г. Ю. В. Линник [57] с помощью созданного им метода большого решета [58] доказал, что гипотеза Виноградова справедлива для почти всех модулей $q$. Более точно теорему Линника можно сформулировать в следующем виде, предложенном С. Учиямой (см. [5; с. 136, с. 217-218]): для $\pi(x)(1-o(1))$ значений $q, q \leqslant x$, наименъший квадратичный невычет $n=n(q)$ и наименьший простой квадратичный вычет $v=v(q)$ не превосходят $c(\varepsilon) \ln ^{2+\varepsilon} x$.

Из оценки Берджесса [24] для $N_{\min }$ получается такое неравенство:

$$
N_{\min } \leqslant q^{\frac{1}{4 \sqrt{e}}+\varepsilon} .
$$

В 1947 г. Ю. В. Линник и А. А. Реньи [59] сформулировали ряд гипотез, обобщающих гипотезу Виноградова, и доказали три своеобразные теоремы относительно этих гипотез. Пусть $\chi(n)$ - неглавный примитивный характер по модулю $D, \pm D$ - фундаментальный дискриминант, $\chi(n)$ - вещественный характер (всюду, кроме теоремы 1). Пусть, далее,

$$
M(\chi)=\max _{1 \leqslant x \leqslant D-1}\left|\sum_{n \leqslant x} \chi(n)\right|, \quad L(1, \chi)=\sum_{n=1}^{\infty} \frac{\chi(n)}{n},
$$

$N_{\min }$ - наименьшее из положительных чисел, для которых $\chi(n) \neq+1 ; N_{\min }^{*}-$ число с наименьшей абсолютной величиной, для которого $\chi(n)=+1 ; P_{\min }-$ наименьшее простое число, для которого $\chi(p)=+1$.

ГиПотезА $1 \mathrm{~A}$.

$$
\lim _{D \rightarrow \infty} \frac{\ln N_{\min }}{\ln D}=0
$$


ГипотезА 1Б.

$$
\lim _{D \rightarrow \infty} \frac{\ln N_{\min }^{*}}{\ln D}=0 .
$$

ГиПотЕЗА 2.

$$
\lim _{D \rightarrow \infty} \frac{\ln P_{\min }}{\ln D}=0 .
$$

ГИПОТЕЗА 3.

$$
\lim _{D \rightarrow \infty} \frac{M(\chi)}{\sqrt{D} \ln D}=0 .
$$

ГИПотеЗА 4.

$$
\lim _{D \rightarrow \infty} \frac{|L(1, \chi)|}{\ln D}=0
$$

Teopema 1.

$$
\lim _{D \rightarrow \infty} \frac{\ln N_{\min }^{*}}{\ln D} \frac{M(\chi)}{\sqrt{D} \ln D}=0 .
$$

TeOpema 2.

$$
\lim _{D \rightarrow \infty} \frac{\ln P_{\min }}{\ln D} \frac{M(\chi)}{\sqrt{D} \ln D}=0 .
$$

Теорема 3. Если выполнено неравенство

$$
\varlimsup_{D \rightarrow \infty} \frac{\ln N_{\min }}{\ln D}>0,
$$

mo

$$
\varlimsup_{D \rightarrow \infty} \frac{|L(1, \chi)|}{\ln D}>0 .
$$

Ни одна из сформулированных гипотез Линника-Реньи к настоящему времени (2008 г.) не доказана. Заметим, что

$$
\sqrt{\frac{D}{12} \prod_{p \mid D}\left(1-\frac{1}{p^{2}}\right)}<M(\chi)<\sqrt{D} \ln D, \quad|L(1, \chi)|<\ln D .
$$

Кроме того, Дж. Литтлвуд [60], предполагая справедливой расширенную гипотезу Римана, доказал, что

$$
\frac{1+o(1)}{2 b \ln \ln D} \leqslant|L(1, \chi)| \leqslant(1+o(1)) \cdot 2 c \ln \ln D,
$$

где $b=\frac{6}{\pi^{2}} e^{\gamma}, \gamma$ - постоянная Эйлера, $c=e^{\gamma}$.

Если $\chi(n)$ - вещественный характер, то по знаменитой теореме Зигеля [61] для любого $\varepsilon>0$ выполняется неравенство:

$$
L(1, \chi) \geqslant c(\varepsilon) D^{-\varepsilon}, \quad c(\varepsilon)>0 .
$$

Замечу, что в [62], [63] Ю. В. Линник аналитическим методом оценивал суммы характеров с простыми числами, а метод большого решета Линника привел к следующей замечательной теореме Бомбьери-Виноградова (см. [64], [65]): при любом $A>0$ найдется $B=B(A)>0$ такое, что

$$
\sum_{Q \leqslant \sqrt{x}(\ln x)^{-B}} \max _{(l, Q)=1}\left|\psi(x ; Q, l)-\frac{x}{\varphi(Q)}\right| \leqslant c(A) x(\ln x)^{-A} .
$$


Оценка Хассе-Вейля (14), которая существенно используется в рассматриваемых вопросах, доказана с помощью методов алгебраической геометрии. И. М. Виноградов неоднократно предлагал найти прямое, чисто арифметическое, доказательство (14).

В 1967 г. в мае месяце на Всесоюзной школе-конференции по алгебре и теории чисел, которая проходила в Таджикистане недалеко от г. Душанбе, И. Р. Шафаревич в своем докладе еще раз обратил внимание на эту проблему. В частности, была поставлена конкретная задача: доказать разрешимость сравнения

$$
y^{2} \equiv x^{3}+a x+b(\bmod q),
$$

не пользуясь методами алгебраической геометрии.

Летом того же года А. Г. Постников нашел элементарный метод, позволяющий оценивать снизу количество решений (28) и, в частности, доказывать разрешимость (28). Метод Постникова состоял в следующем (см. [66], [5; с. 213]). Пусть $q \geqslant 5, f(x)=x^{3}+a x+b$. Рассмотрим многочлены

$$
F(x)=1 \pm(f(x))^{\frac{q-1}{2}}, \quad g(x)=2 f(x)\left(1 \pm(f(x))^{\frac{q-1}{2}}\right)+f^{\prime}(x)\left(x^{q}-x\right) .
$$

Легко видеть, что каждый нуль $F(x)$ по модулю $q$ является по крайней мере двукратным нулем $g(x)$. Пусть, далее, $N$ - число решений $(28), j_{1}, j_{2}, j_{0}-$ числа решений сравнений

$$
1+(f(x))^{\frac{q-1}{2}} \equiv 0(\bmod q), \quad 1-(f(x))^{\frac{q-1}{2}} \equiv 0(\bmod q), \quad f(x) \equiv 0(\bmod q)
$$

соответственно. Очевидно, что $N=2 j_{2}+j_{0}$, и, кроме того, $j_{1}+j_{2}+j_{0}=q$,

$$
2 j_{1}+j_{0} \leqslant \operatorname{deg} g(x)=\frac{3}{2}(q+1), \quad 2 j_{2}+j_{0} \leqslant \operatorname{deg} g(x)=\frac{3}{2}(q+1) .
$$

Отсюда получаем:

$$
|N-q| \leqslant \frac{q+3}{2}, \quad N \geqslant \frac{q-3}{2}>0
$$

Естественно было предположить, что если строить аналоги $g(x)$, у которых каждый нуль $F(x)$ является нулем высокой кратности, то оценка (29) может быть уточнена. Эти предположения были подтверждены С. А. Степановым, который в работах [67]-[70] получил элементарным методом оценки Хассе-Вейля как для сумм мультипликативных, так и для сумм аддитивных характеров в конечных полях (см. также [71]).

Исследования Степанова были продолжены Н. М. Коробовым [72], который для суммы символов Лежандра получил оценку более точную, чем (14):

$$
\left|\sum_{x=1}^{q}\left(\frac{f(x)}{q}\right)\right| \leqslant(n-1) \sqrt{q-\frac{(n-3)(n-4)}{4}} .
$$

Здесь $n$ - нечетное число, $n \geqslant 3$,

$$
f(x)=a_{0}+a_{1} x+\cdots+a_{n} x^{n},
$$


$a_{0}, a_{1}, \ldots, a_{n}$ - целые числа, $\left(a_{n}, q\right)=1$ (см. также работу [73] Д. А. Митькина, в которой конструкция Коробова использована при оценках рациональных тригонометрических сумм).

Метод $\S 3$ главы II был применен М. М. Петечуком [74] к проблеме асимптотического поведения суммы $S(x)$,

$$
S(x)=\sum_{\substack{n \leqslant x \\ n \equiv l(\bmod D)}} \tau_{k}(n) .
$$

Проблема состоит в том, чтобы получать асимптотики $S(x)$ при возможно бо́льших значениях $D=D(x)$. Пользуясь, сверх того, оценкой А. Г. Постникова [75] короткой суммы характеров, М. М. Петечук для модулей $D$, равных степени фиксированного простого числа, получил асимптотику $S(x)$ при условии, что $D \leqslant x^{3 / 8-\varepsilon}$. В это же время и этим же методом М. М. Петечук получил асимптотику $S(x)$ при любых $D$, причем $D$ были много больше, чем $D$ в соответствующей теореме А. Ф. Лаврика [76], доказанной аналитическим методом. K сожалению, этот результат так и не был опубликован М. М. Петечуком.

Оценки сумм характеров с простыми числами были использованы 3. Х. Рахмоновым в проблеме наименьшего гольдбахова числа и числа Харди-Литтлвуда в арифметической прогрессии, в проблеме средних значений функции Чебышёва $\psi(x, \chi)($ см. [77]-[83]). К этой же проблематике относятся оценки сумм характеров по бесквадратным числам, состоящим из заданного числа простых сомножителей (см. [84], [85], а также статьи Г. Д. Негматовой [86], [87]).

Применение оценок сумм характеров и метода $\S 3$ гл. II позволило М. З. Гараеву и автору [88] доказать теорему о том, что для почти всех $l$ по модулю $q$ разрешимо сравнение

$$
x y \equiv l(\bmod q),
$$

где $\max (x, y) \leqslant q^{5 / 8+\varepsilon}, q \geqslant q_{1}(\varepsilon)$. В этой же статье доказано следующее неравенство:

$$
\left|\sum_{n \leqslant x} \chi(n)\right| \ll x^{\frac{1}{r}} q^{\frac{1}{2}-\frac{3}{4 r}+\frac{1}{4 r^{2}}+\varepsilon} .
$$

Здесь $r$ - произвольное натуральное число, постоянная в знаке « зависит только от $r$ и $\varepsilon$. Раньше это неравенство условно было доказано А. И. Виноградовым [89]. А.И. Виноградов пользовался тем, что при любом целом числе $a$, $0 \leqslant a<q$, выполняется оценка:

$$
\left|\sum_{B<n \leqslant B+A} \chi(n) e^{2 \pi i \frac{a n}{q}}\right| \ll A^{1-\frac{1}{r}} q^{\frac{1}{4 r}+\frac{1}{4 r^{2}}+\varepsilon} .
$$

Замечу, что М.З. Гараев и автор [90] другим методом доказали, что для почти всех $l$ по модулю $q$ указанное сравнение разрешимо при $\max (x, y) \leqslant$ $\sqrt{q}(\ln q)^{1+\varepsilon}$.

Результаты главы III для многочленов $f(x)$ произвольной степени и для $\chi(n)$, равных символу Лежандра, условно доказаны в [34]. Результаты П. Делиня [91] 
позволяют надеяться, что справедлива следующая ГипотЕзА: ecли $f(x)-$ многочлен с иелыми коэфбициентами, не являющийся полным квадратом по модулю $q$, целье числа $a_{1}, \ldots, a_{k}$ попарно не сравнимы по модулю $q, k \geqslant 2$,

$$
F(x, y)=f\left(x+a_{1} y\right) \cdots f\left(x+a_{k} y\right),
$$

то справедлива оценка

$$
\left|\sum_{x=1}^{q} \sum_{y=1}^{q}\left(\frac{F(x, y)}{q}\right)\right| \ll q,
$$

где постоянная в знаке « зависит только от $k$ и степени $f(x)$. Для многочлена $f(x)=(x+a)(x+b), a \not \equiv b(\bmod q)$, эта гипотеза справедлива. С помощью неравенства (30) в [34] доказано, что если $X \gg q^{0.75+\omega}, 0<\omega<1 / 2$, mo

$$
\left|\sum_{p \leqslant X}\left(\frac{f(p)}{q}\right)\right| \ll \pi(X) q^{-0.01 \omega^{2}} .
$$

Своеобразное применение оценок сумм характеров к проблеме распределения простых чисел в квазипрогрессиях содержится в работе С.М. Воронина и Р. Турганалиева [92].

Суммы характеров по натуральным числам, имеющим либо только четное, либо только нечетное количество единиц при записи их в двоичной системе счисления, были рассмотрены автором и Б. Новаком в [93]. Пусть $n=\alpha_{0}+$ $\alpha_{1} \cdot 2+\cdots+\alpha_{k} \cdot 2^{k}$, где $\alpha_{j}=0$ или $1, j=0,1, \ldots, k$, и при $\nu=0,1$ символом $E_{\nu}$ обозначим множество натуральных чисел $n$, для которых

$$
\alpha_{0}+\alpha_{1}+\cdots+\alpha_{k} \equiv \nu(\bmod 2) .
$$

В [93] доказана тЕоремА: nусть $\lambda=1-\frac{\ln 3}{\ln 4}=0.2075187496 . .$, , u nyсmъ

$$
\Delta=\Delta(X ; q)= \begin{cases}\sqrt{X^{-1} \sqrt{q} \ln q}, & \text { если } \sqrt{q} \ln q<X \leqslant(\sqrt{q})^{5} \ln q \\ q^{-1 / 2}, & \text { если }(\sqrt{q})^{5} \ln q<X \leqslant(\sqrt{q})^{4+1 / \lambda} \\ \left(X q^{-2}\right)^{-\lambda}, & \text { если }(\sqrt{q})^{4+1 / \lambda}<X .\end{cases}
$$

Тогда для $S_{\nu}(X)$

$$
S_{\nu}(X)=\sum_{\substack{n \leqslant X \\ n \in E_{\nu}}} \chi(n),
$$

справедлива оценка

$$
\left|S_{\nu}(X)\right| \ll X \Delta
$$

При доказательстве этой теоремы авторы существенно пользовались оценками К. М. Эминяна [94]-[96]. Арифметическими следствиями теоремы являются следующие асимптотические формулы:

а) если $m \mid(q-1), m \geqslant 2, K_{m, \nu}(X)$ - количество вычетов степени $m$ по модулю q в последовательности чисел $n \leqslant X, n \in E_{\nu}$, то

$$
K_{m, \nu}(X)=\frac{1}{2 m} X\left(1+\Delta_{1}\right)
$$


где

$$
\left|\Delta_{1}\right| \ll m \Delta+X^{-\lambda}+q^{-1} ;
$$

б) если $k$ - количество простых делителей числа $q-1$, a $T_{\nu}(X)$ - количество первообразных корней по модулю $q$, не превосходящих $X$ и принадлежащих $E_{\nu}$, mo

$$
T_{\nu}(X)=\frac{\varphi(q-1)}{q-1} X\left(1+\Delta_{2}\right),
$$

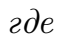

$$
\left|\Delta_{2}\right| \ll X^{-\lambda} \ln ^{2} X+q^{-1} \ln ^{2} X+\Delta \cdot 2^{k} \ln X .
$$

И, наконец, несколько необычной является теорема, доказанная в [97]: существуют бесконечная последовательность чисел $q$ и последовательность многочленов вида $x^{n}+a$, где $(a, q)=1$ и

$$
4 \frac{q-1}{\ln q} \leqslant n \leqslant 8 \frac{q-1}{\ln q},
$$

такие, что выполняется следующее равенство:

$$
\left|\sum_{x=1}^{q}\left(\frac{x^{n}+a}{q}\right)\right|=q .
$$

Среди нерешенных проблем в первую очередь следует назвать проблемы-гиnотезы, поставленные Виноградовым и Линником-Реньи. Другими проблемами являются такие:

1. Доказать оценку леммы 3 главы II при $M \geqslant q^{c+\gamma}$ и $c<1 / 4$.

2. Доказать оценку теоремы 1 главы II при $X \geqslant q^{c+\omega}$ и $c<1 / 2$.

3. Доказать оценку теоремы 1 главы III при $X \geqslant q^{c+\omega}$ и $c<3 / 4$.

4. Доказать неравенство (30).

5. Хотя бы в одной из оценок, которые получены в теоремах 1,2 и лемме 3 главы II, теоремах 1, 2 главы III, теоремах 1-5 главы IV, теореме 5 главы V, понижающий множитель, который имеет вид $q^{-0.02 \omega^{2}}$, либо $q^{-0.5 \gamma^{2}}$, либо $q^{-0.02 \delta^{2}}$, заменить на понижающий множитель вида $q^{-c \omega^{\alpha}}, q^{-c \gamma^{\alpha}}, q^{-c \delta^{\alpha}}$ соответственно, где $c$ и $\alpha$ - абсолютные положительные постоянные, причем $\alpha<2$.

6. Доказать, что при любых $A$ и $B$ с условием $A \subset[1, q-1], B \subset[1, q-1]$, $\|A\| \asymp \sqrt{q},\|B\| \asymp \sqrt{q}$, для суммы $W$,

$$
W=\sum_{x \in A} \sum_{y \in B} \chi(x+y),
$$

справедлива оценка

$$
|W| \leqslant c(\delta)\|A\|\|B\| q^{-\delta}, \quad \delta>0 .
$$

7. Доказать существование бесконечной последовательности простых чисел $q$ и бесконечной последовательности бесквадратных по модулю $q$ многочленов $f(x)$ степени $n=o\left(\frac{q}{\ln q}\right)$, например, $n=O\left(\frac{q}{\ln q \ln \ln q}\right)$, для которых выполняется равенство

$$
\left|\sum_{x=1}^{q}\left(\frac{f(x)}{q}\right)\right|=q .
$$


8. В [98] А. О. Гельфонд дал элементарное доказательство теоремы Пейджа в следующей формулировке: если ұ - вещественный первообразный характер по модулю $m$, то справедливо неравенство:

$$
|L(1, \chi)|>\frac{1}{33 \sqrt{m} \ln ^{2} m}, \quad m>m_{0} .
$$

При получении (31) А. О. Гельфонд пользовался только оценкой ВиноградоваПойя (см. также [99; с. 281], [47; с. 123-124]). Нельзя ли методом Гельфонда (или его вариантами) получить более сильную оценку, чем (31)?

\section{Список литературы}

[1] П. Г. Л. Дирихле, Лекции по теории чисел, ОНТИ, М.-Л., 1936; англ. пер. с нем. ориг. 1983 г.: P. G. L. Dirichlet, Lectures on number theory, History of Mathematics, 16, Amer. Math. Soc. / London Math. Soc., Providence, RI / London, 1999.

[2] И. М. Виноградов, Основы теории чисел, Наука, М., 1981.

[3] Г. Дэвенпорт, Мультипликативная теория чисел, Наука, М., 1971; пер. с англ.: H. Davenport, Multiplicative number theory, Lectures in Advanced Mathematics, 1, Markham, Chicago, IL, 1967.

[4] А. А. Карацуба, "Суммы характеров на последовательности сдвинутых простых чисел и их применения", Матем. заметки, 17:1 (1975), 155-159; англ. пер.: A. A. Karatsuba, "Sums of characters in sequences of shifted prime numbers, with applications", Math. Notes, 17 (1975), 91-93.

[5] А. А. Карацуба, Основы аналитической теории чисел, Наука, М., 1983, 2-изд., перераб. и доп.; англ. пер.: [48].

[6] Р. Лидл, Г. Нидеррайтер, Конечные поля, т. I, II, Мир, М., 1988; пер. с англ.: R. Lidl, H. Niederreiter, Finite fields, Encyclopedia Math. Appl., 20, Addison-Wesley, Reading, MA, 1983.

[7] Г. Монтгомери, Мультипликативная теория чисел, Мир, М., 1974; пер. с англ. H. L. Montgomery, Topics in multiplicative number theory, Lecture Notes in Math., 227, Springer-Verlag, Berlin-New York, 1971.

[8] K. Прахар, Распределение простых чисел, Мир, М., 1967; пер. с нем.: К. Prachar, Primzahlverteilung, Grundlehren Math. Wiss., 91, Springer-Verlag, BerlinGöttingen-Heidelberg, 1957.

[9] Г. Хассе, Лекции по теории чисел, ИЛ, М., 1953; пер. с нем.: Н. Hasse, Vorlesungen über Zahlentheorie, Die Grundlehren der mathematischen Wissenschaften in Einzeldarstellungen mit besonderer Berücksightigung der Anwendungsgebiete, 59, Springer-Verlag, Berlin-Göttingen-Heidelberg, 1950.

[10] Н. Г. Чудаков, Введение в теорию L-функиий Дирихле, ОГИЗ, М.-Л., 1947.

[11] И. М. Виноградов, "Sur la distribution des residus et des nonresidus des puissances", Журн. физ.-матем. об-ва при Пермском ун-те, 1918, № 1, 94-98.

[12] И. М. Виноградов, "Об одном асимптотическом равенстве теории квадратичных форм", Журн. физ.-матем. об-ва при Пермском ун-те, 1918, № 1, 18-28.

[13] G. Pólya, "Über die Verteilung der quadratischen Reste und Nichtreste", Nachr. Akad. Wiss. Göttingen Math.-Phys. Kl. II, 1918, 21-29.

[14] H. Davenport, "On primitive roots in finite fields", Quart. J. Math., 8 (1937), 308-312.

[15] L. Carlitz, S. Uchiyama, "Bounds for exponential sums", Duke Math. J., 24:1 (1957), $37-41$.

[16] Г.И. Перельмутер, "О некоторых суммах с характерами", УМН, 18:2 (1963), $145-149$. 
[17] И. М. Виноградов, "Распределение квадратичных вычетов и невычетов вида $p+k$ по простому модулю", Матем. сб., 3:2 (1938), 311-320.

[18] И. М. Виноградов, "Уточнение метода оценки сумм с простыми числами", Изв. АН СССР. Сер. матем., 7:1 (1943), 17-34.

[19] И. М. Виноградов, "Новый подход к оценке суммы значений $\chi(p+k)$ ", Изв. АН СССР. Сер. матем., 16:3 (1952), 197-210.

[20] Ю.В. Линник, "Новейшие работы И. М. Виноградова", Труды международной конференции по теории чисел (Москва, 1971), Тр. МИАН, 132, Наука, М., 1973, $27-29$.

[21] А. А. Карацуба, "Распределение пар квадратичных вычетов и невычетов специального вида", Изв. АН СССР. Сер. матем., 51:5 (1987), 994-1009; англ. пер.: A. A. Karatsuba, "The distribution of pairs of quadratic residues and nonresidues of a special form", Math. USSR-Izv., 31:2 (1988), 307-323.

[22] И. М. Виноградов, "Улучшение оценки для суммы значений $\chi(p+k)$ ", Изв. АН СССР. Сер. матем., 17:4 (1953), 285-290.

[23] Г. И. Перельмутер, "Оценка одной суммы с простыми числами”, Докл. АН CCCP, 144:1 (1962), 48-51; англ. пер.: G. I. Perel'muter, "Evaluation of a sum containing primes", Soviet Math. Dokl., 3 (1962), 663-667.

[24] D. A. Burgess, "The distribution of quadratic residues and non-residues", Mathematika, 4:8 (1957), 106-112.

[25] D. A. Burgess, "On character sums and primitive roots", Proc. London Math. Soc. (3), 12:1 (1962), 179-192.

[26] D. A. Burgess, "On character sums and L-series", Proc. London Math. Soc. (3), 12:1 (1962), 193-206.

[27] D. A. Burgess, "On character sums and L-series. II", Proc. London Math. Soc. (3), 13:1 (1963), 524-536.

[28] D. A. Burgess, "Character sums and primitive roots in finite fields", Proc. London Math. Soc. (3), 17:1 (1967), 11-25.

[29] H. Davenport, D. J. Lewis, "Character sums and primitive roots in finite fields", Rend. Circ. Mat. Palermo (2), 12:2 (1963), 129-136.

[30] А. А. Карацуба, "Суммы характеров и первообразные корни в конечных полях", Докл. АН СССР, 180:6 (1968), 1287-1289; англ. пер.: А. A. Karatsuba, "Character sums and primitive roots in finite fields", Soviet Math. Dokl., 9 (1968), 755-757.

[31] А. А. Карацуба, "Об оценках сумм характеров", Изв. АН СССР. Сер. матем., 34:1 (1970), 20-30; англ. пер.: А. А. Karatsuba, "Estimates of character sums", Math. USSR-Izv., 4:1 (1970), 19-29.

[32] А.А. Карацуба, "О суммах характеров с простыми числами", Докл. АН СCCP, 190:3 (1970), 517-518; англ. пер.: А. А. Karatsuba, "On sums of characters with primes", Soviet Math. Dokl., 11 (1970), 135-137.

[33] А. А. Карацуба, "Суммы характеров с простыми числами", Изв. АН СССР. Сер. матем., 34:2 (1970), 299-321; англ. пер.: A. A. Karatsuba, "Sums of characters over prime numbers", Math. USSR-Izv., 4:2 (1970), 303-326.

[34] А.А. Карацуба, "Распределение значений символов Лежандра от многочленов с простыми числами", Докл. АН СССР, 238:3 (1978), 524-526; англ. пер.: A. A. Karatsuba, "The distribution of values of the Legendre symbols of polynomials with prime numbers", Soviet Math. Dokl., 19:1 (1978), 61-63.

[35] А. А. Карацуба, "Суммы символов Лежандра от многочленов второй степени с простыми числами", Изв. АН СССР. Сер. матем., 42:2 (1978), 315-324; англ. пер.: A. A Karatsuba, "Sums of Legendre symbols of polynomials of second degree over prime numbers", Math. USSR-Izv., 12:2 (1978), 299-308. 
[36] Г. Пойя, Г. Сегё, Задачи и теоремъ из анализа, ч. І, ГИТТЛ, М., 1956; пер. с нем.: G. Pólya, G. Szegö, Aufgaben und Lehrsätze aus der Analysis, vol. I: Reihen, Integralrechnung, Funktionentheorie, Die Grundlehren der mathematischen Wissenschaften in Einzeldarstellungen mit besonderer Berücksichtigung der Anwendungsgebiete, 19, Springer-Verlag, Berlin-Göttingen-Heidelberg, 1954.

[37] И. М. Виноградов, "Оценка одной суммы, распространенной на простые числа арифметической прогрессии”, Изв. АН СССР. Сер. матем., 30:3 (1966), 481-496.

[38] А.А. Карацуба, "Суммы характеров с простыми числами, принадлежащими арифметической прогрессии", Изв. АН СССР. Сер. матем., 35:3 (1971), 469-484; англ. пер.: А. А. Karatsuba, "Sums of characters with prime numbers in an arithmetic progression", Math. USSR-Izv., 5:3 (1971), 485-501.

[39] А. А. Карацуба, "Квадратичные вычеты и невычеты в редких последовательностях", Докл. АН ССCP, 279:5 (1984), 1044-1046; англ. пер.: А. A. Karatsuba, "Quadratic residues and nonresidues in sparse sequences", Soviet Math. Dokl., 30:3 (1984), 767-768.

[40] А.А. Карацуба, "Распределение степенных вычетов и невычетов в аддитивных последовательностях", Докл. АН СCCP, 196:4 (1971), 759-760; англ. пер.: A. A. Karatsuba, "Distribution of power residues and non-residues in additive sequences", Soviet Math. Dokl., 12 (1971), 235-235.

[41] А.А. Карацуба, "Распределение значений характеров Дирихле на аддитивных последовательностях", Докл. АН СССР, 319:3 (1991), 543-544; англ. пер.: A. A. Karatsuba, "The distribution of values of Dirichlet characters on additive sequences", Soviet Math. Dokl., 44:1 (1992), 145-148.

[42] H. Davenport, P. Erdös, "The distribution of quadratic and higher residues", Publ. Math. Debrecen, 2 (1952), 252-265.

[43] P. Erdös, H. N. Shapiro, "On the least primitive roots of a prime", Pacific J. Math., 7:1 (1957), 861-865.

[44] А.А. Карацуба, "Об одной арифметической сумме", Докл. АН CCCP, 199:4 (1971), 770-772; англ. пер.: А. А. Karatsuba, "On a certain arithmetic sum", Soviet Math. Dokl., 12 (1971), 1172-1174.

[45] А. А. Карацуба, "Асимптотика одной арифметической суммы”, Матем. заметки, 24:6 (1978), 737-740; англ. пер.: А. A. Karatsuba, "The asymptotics of a certain arithmetical sum", Math. Notes, 24:5-6 (1978), 893-895.

[46] А.А. Карацуба, "Суммы характеров с весами", Изв. РАН. Сер. матем., 64:2 (2000), 29-42; англ. пер.: А. А. Karatsuba, "Weighted character sums", Izv. Math., 64:2 (2000), 249-263.

[47] А. А. Карацуба, Основы аналитической теории чисел, Наука, М., 1975.

[48] A. A. Karatsuba, Basic analytic number theory, Springer-Verlag, Berlin, 1993; пер. с русск.: [5].

[49] A. A. Karatsuba, "Sums of characters with prime numbers and their applications", Tatra Mt. Math. Publ., 20 (2000), 155-162.

[50] И. М. Виноградов, Избранные труды, Изд-во АН СССР, М., 1952.

[51] И. М. Виноградов, Особые варианты метода тригонометрических сумм, Наука, M., 1976.

[52] Э.К. Фогелс, "О простых числах в начале арифметической прогрессии", Докл. AH CCCP, 102:3 (1955), 455-456.

[53] U. V. Linnik, "On the least prime in an arithmetic progression. I: The basic theorem", Матем. сб., 15:2 (1944), 139-178; "On the least prime in an arithmetic progression. II: The Deuring-Heilbronn theorem", Матем. сб., 15:3 (1944), 347-368.

[54] А. А. Карацуба, "Распределение произведений сдвинутых простых чисел в арифметических прогрессиях", Докл. АН СССР, 192:4 (1970), 724-727; англ. пер.: 
A. A. Karatsuba, "The distribution of products of shifted primes in arithmetical progressions", Soviet Math. Dokl., 11 (1970), 707-711.

[55] И. М. Виноградов, "О границе наименьшего невычета $n$-й степени", Изв. АН CCCP, сер. VI, 20:1-2 (1926), 47-58.

[56] И. М. Виноградов, "О распределении квадратичных вычетов и невычетов", Журн. физ.-матем. об-ва при Пермском ун-те, 1919, № 2, 1-16.

[57] Ю.В. Линник, "Замечание о наименьшем квадратичном невычете", Докл. АН CCCP, 36:4-5 (1942), 119-120.

[58] Ю. В. Линник, "Большое решето", Докл. АН СССР, 30:4 (1941), 292-294.

[59] Ю. В. Линник, А. А. Реньи, "О некоторых гипотезах теории характеров Дирихле", Изв. АН СССР. Сер. матем., 11:6 (1947), 539-546.

[60] J. E. Littlewood, "On the class-number of the corpus $P(\sqrt{-k})$ ", Proc. London Math. Soc. (2), 27:1 (1928), 358-372.

[61] C. L. Siegel, "Über die Klassenzahl quadratischer Zahlkörper", Acta Arith., 1 (1935), 83-86.

[62] Ю.В. Линник, "О распределении характеров", Докл. АН СССР, 42:8 (1944), 323-325.

[63] Ю. В. Линник, “О характерах простых чисел. I”, Матем. сб., 16:2 (1945), 101-120.

[64] E. Bombieri, "On the large sieve", Mathematika, 12 (1965), 201-225.

[65] А.И. Виноградов, "О плотностной гипотезе для $L$-рядов Дирихле", Изв. $A H$ СССР. Сер. матем., 29:4 (1965), 903-934.

[66] Л. П. Постникова, Тригонометрические суммы и теория сравнений по простому модулю, Учебное пособие МГПИ им. В. И. Ленина, 1973.

[67] С. А. Степанов, "О числе точек гиперэллиптической кривой над простым конечным полем”, Изв. АН СССР. Сер. матем., 33:5 (1969), 1171-1181; англ. пер.: S. A. Stepanov, "On the number of points of a hyperelliptic curve over a finite prime field", Math. USSR-Izv., 3:5 (1969), 1103-1114.

[68] S. A. Stepanov, "Elementary method in the theory of congruences for a prime modulus", Acta Arith., 17 (1970), 231-247.

[69] С. А. Степанов, "Об оценке рациональных тригонометрических сумм с простым знаменателем", Сб. статей, посв. акад. И. М. Виноградову к его 80-летию, Тр. МИАН, 112, Наука, М., 1971, 346-371.

[70] S. A. Stepanov, "An elementary proof of the Hasse-Weil theorem for hyperelliptic curves", J. Number Theory, 4:2 (1972), 118-143.

[71] С. А. Степанов, Арифметика алгебраических кривых, Наука, М., 1991; англ. пер.: S. A. Stepanov, Arithmetic of algebraic curves, Monogr. Contemp. Math., Consultants Bureau, New York, 1994.

[72] Н. М. Коробов, "Оценка суммы символов Лежандра", Докл. АН CCCP, 196:4 (1971), 764-767; англ. пер.: N. M. Korobov, "Estimate of a sum of Legendre symbols", Soviet Math. Dokl., 12 (1971), 241-245.

[73] Д.А. Митькин, "Об элементарном доказательстве оценки А. Вейля для рациональных тригонометрических сумм с простым знаменателем", Изв. вузов. Матем., 1986, № 6, 14-17; англ. пер.: D. A. Mit’kin, "An elementary proof of A. Weil’s estimate for rational trigonometric sums with a prime denominator", Soviet Math., 30:6 (1986), 18-23.

[74] М. М. Петечук, "Сумма значений функции делителей в арифметических прогрессиях с разностью, равной степени нечетного простого числа", Изв. АН СССР. Сер. матем., 43:4 (1979), 892-908; англ. пер.: М. M. Petečuk, "The sum of the values of the divisor function in arithmetic progressions whose difference is a power of an odd prime", Math. USSR-Izv., 15:1 (1980), 145-160.

[75] А. Г. Постников, "О сумме характеров по модулю, равному степени простого числа", Изв. АН СССР. Сер. матем., 19:1 (1955), 11-16. 
[76] А.Ф. Лаврик, "Функциональное уравнение для $L$-функций Дирихле и задача делителей в арифметических прогрессиях", Изв. АН СССР. Сер. матем., 30:2 (1966), 433-448.

[77] З.Х. Рахмонов, "О распределении значений характеров Дирихле", УМH, 41:1 (1986), 201-202; англ. пер.: Z. Kh. Rakhmonov, "On the distribution of values of Dirichlet characters", Russian Math. Surveys, 41:1 (1986), 237-238.

[78] 3.Х. Рахмонов, "Об оценке сумм характеров с простыми числами", Докл. АН ТаджСCP, 29:1 (1986), 16-20.

[79] 3. Х. Рахмонов, "О наименьшем Гольдбаховом числе в арифметической прогрессии", Изв. АН ТаджССР. Отд. физ.-матем. и геол. наук, 1986, № 2(100), 103-106.

[80] 3. Х. Рахмонов, "Распределение чисел Харди-Литтлвуда в арифметических прогрессиях", Изв. АН СССР. Сер. матем., 53:1 (1989), 211-224; англ. пер.: Z. Kh. Rakhmonov, "The distribution of Hardy-Littlewood numbers in arithmetic progressions", Math. USSR-Izv., 34:1 (1990), 213-228.

[81] З.Х. Рахмонов, "Теорема о среднем значении $\psi(x, \chi)$ и ее приложения", Изв. PAH. Сер. матем., 57:4 (1993), 55-71; англ. пер.: Z. Kh. Rakhmonov, "Theorem on the mean value of $\psi(x, \chi)$ and its applications", Russian Acad. Sci. Izv. Math., 43:1 (1994), 49-64.

[82] З.Х. Рахмонов, "Средние значения функции Чебышёва", Докл. РАН, 331:3 (1993), 281-282; англ. пер.: Z. Kh. Rakhmonov, "Mean values of the Chebyshev function", Russian Acad. Sci. Dokl. Math., 48:1 (1994), 85-87.

[83] З.Х. Рахмонов, “Теорема о среднем значении функций Чебышёва”, Изв. РАН. Сер. матем., 58:3 (1994), 127-139; англ. пер.: Z. Kh. Rakhmonov, "A theorem on the mean value of Chebyshev functions", Russian Acad. Sci. Izv. Math., 44:3 (1995), $555-569$.

[84] И. М. Виноградов, "О распределении произведений простых чисел и значений функции Мёбиуса", Изв. АН СССР. Сер. матем., 12:4 (1948), 341-350.

[85] А. А. Карацуба, "О распределении значений неглавных характеров", Тр. МИАН, 142, Наука, М., 1976, 156-164; англ. пер.: A. A. Karatsuba, "On the distribution of values of nonprincipal characters", Proc. Steklov Inst. Math., 142 (1979), 165-174.

[86] Г. Д. Негматова, "Распределение степенных вычетов в редких последовательностях", Докл. АН ТаджССР, 28:4 (1985), 193-197.

[87] Г. Д. Негматова, "Распределение значений неглавных характеров в редких последовательностях", УМH, 44:5 (1989), 177-178; англ. пер.: G. D. Negmatova, "The distribution of values of non-principal characters in lacunary sequences", Russian Math. Surveys, 44:5 (1989), 214-215.

[88] M.Z. Garaev, A.A. Karatsuba, "On character sums and the exceptional set of a congruence problem", J. Number Theory, 114:1 (2005), 182-192.

[89] А.И. Виноградов, "О свойстве симметрии сумм характеров Дирихле", Изв. АН УзбССР. Сер. физ.-матем., 9:1 (1965), 21-27.

[90] M. Z. Garaev, A. A. Karatsuba, "The representation of residue classes by products of small integers", Proc. Edinb. Math. Soc. (2), 50:2 (2007), 363-375.

[91] P. Deligne, "La conjecture de Weil. I", Inst. Hautes Études Sci. Publ. Math., 43:1 (1974), 273-307; "La conjecture de Weil. II", 52:1 (1980), 137-252.

[92] С. М. Воронин, Р. Турганалиев, "О простых числах в квазипрогрессиях", Вестн. АН Каз СCP, 1988, № 6, 47-53.

[93] А. А. Карацуба, Б. Новак, “Арифметические задачи с числами специального вида", Матем. заметки, 66:2 (1999), 315-317; англ. пер.: А. A. Karatsuba, B. Novak, "Arithmetical problems with numbers of special type", Math. Notes, 66:2 (1999), 251-253. 
[94] К. М. Эминян, "О проблеме делителей Дирихле в некоторых последовательностях натуральных чисел", Изв. АН СССР. Сер. матем., 55:3 (1991), 680-686; англ. пер.: K. M. Èminyan, "On the Dirichlet divisor problem in some sequences of natural numbers", Math. USSR-Izv., 38:3 (1992), 669-675.

[95] К. М. Эминян, “О представлении чисел с заданными свойствами двоичного разложения суммами двух квадратов", Теория чисел и анализ: Труды международной конференции, посв. 100-летию со дня рождения акад. И. М. Виноградова, Тр. МИАН, 207, Наука, М., 1994, 377-382; англ. пер.: K. M. Ėminyan, "On the representation of numbers with given properties of binary expansion by sums of two squares", Proc. Steklov Inst. Math., 207 (1995), 347-351.

[96] К. М. Эминян, "Об одной бинарной задаче", Матем. заметки, 60:4 (1996), 634-637; англ. пер.: K. M. Ėminyan, "A binary problem”, Math. Notes, 60:4 (1996), $478-481$.

[97] А. А. Карацуба, "Об оценках снизу сумм характеров от многочленов", Матем. заметки, 14:1 (1973), 67-72; англ. пер.: A. A. Karatsuba, "Lower bounds for sums of the characters of polynomials", Math. Notes, 14 (1973), 593-596.

[98] А. О. Гельфонд, "Об одном элементарном подходе к некоторым задачам из области распределения простых чисел”, Вестн. МГУ, сер. физ.-матем. и естеств. наук, вып. 1, № 2, 1953, 21-26.

[99] А. О. Гельфонд, Избранные труды, Наука, М., 1973.

А. А. Карацуба (А. А. Karatsuba)

Математический институт им. В. А. Стеклова РАН

E-mail: karatsuba@mi.ras.ru
Поступила в редакцию 10.03 .2008 